Pacific Journal of Mathematics

ON THE STRUCTURE OF FINITELY GENERATED SPLITTING 


\title{
ON THE STRUCTURE OF FINITELY GENERATED SPLITTING RINGS
}

\author{
John Fuelberth AND JAmes KuZMANOviCH
}

In this paper the structure of finitely generated splitting rings for the Goldie theory is studied. First, right nonsingular finitely generated splitting rings with essential socle which either are right finite dimensional or are right orders in a semiprimary ring are characterized. This characterization is in terms of an explicit triangular matrix structure for $R$. Then right nonsingular finitely generated splitting rings with zero socle are shown to be right finite dimensional if and only if they are right orders in a semiprimary ring. An explicit triangular structure is given for this class of rings as well. For certain classes of right nonsingular right finite dimensional finitely generated splitting rings with zero socle, the structure theorem can be simplified somewhat. Then right nonsingular right finite dimensional finitely generated splitting rings are characterized as a certain essential product of a ring with essential socle and one with zero socle. Right nonsingular finitely generated splitting rings which are right orders in a semiprimary ring are shown to be a direct product of a ring with essential socle and a ring with zero socle. Finally, some comments are made showing how some of these results can be applied to bounded splitting rings and splitting rings.

1. Preliminaries. In this paper all rings $R$ are associative with identity and all $R$-modules are unital. Unless indicated otherwise, all modules are right modules. A left or right $R$-module $M$ will be denoted by ${ }_{i} M$ or $M_{R}$ respectively. The socle of an $R$ module $M$ will be denoted by soc $(M)$; the socle of $R$ will always mean the socle of $R_{R}$ unless indicated otherwise.

If $M$ is a right $R$-module and $X \subseteq M$, then the right annihilator of $X$ is denoted by $r_{n}(X)$ or $r(X)$ if there is no ambiguity. Similarly for a left $R$-module $M$ and $X \leqq M, l_{R}(X)$ is the left annihilator of $X$.

A submodule $K$ of an $R$-module $M$ is an essential submodule of $M$ if $K \cap L \neq 0$ for all nonzero submodules $L$ of $M$. A right (left) ideal $I$ of $R$ is essential in $R$ if $I$ is essential in $R_{R}\left({ }_{R} R\right)$. Let . $(R)$ denote the family of all essential right ideals of $R$. For any right $R$-module $M, Z(M)=\{x \in M: r(x) \in \mathscr{S}(R)\}$ is called the singular submodule of $M$. The singular submodule of a left $R$-module is defined similarly. An $R$-module $M$ is singular if $Z(M)=M$; $M$ is nonsingular if $Z(M)=0$. A ring $R$ is right (left)-nonsingular if 
$\left.R_{R}{ }_{R} R\right)$ is a nonsingular module.

A submodule $A$ of a module $B$ is closed in $B$ provided that $B / A$ is nonsingular, and we let $L^{*}(B)$ denote the collection of all closed submodules of $B$. For any submodule $A$ of $B$, there is a smallest closed submodule $C$ in $B$ which contains $A$, called the closure of $A$ in $B$. The closure of a two-sided ideal of $R$ is a two-sided ideal of $R$. By a "two-sided ideal of $L^{*}(R)$ " we will mean a two-sided ideal of $R$ belonging to $L^{*}(R)$. We use $S^{0}$ to stand for the localization functor associated with a right nonsingular ring: $S^{0} R$ is the maximal right quotient ring and $S^{0} R$ is a regular self-injective ring.

The $R$-module $M$ is of finite (Goldie) dimension over $R$ if every direct sum of nonzero submodules of $M$ contains only a finite number of summands. We say that $R$ has right finite (Goldie) dimension if $R_{R}$ has finite (Goldie) dimension. If $R$ is a right nonsingular right finite dimensional ring, then $S^{0} R$ is semisimple Artinian and $S^{\circ} R$ is left flat by [26].

Any hereditary torsion theory $(\mathscr{T}, \mathscr{F})$ corresponds to an idempotent kernel functor $\sigma$. (See [12], [16] or [30] for the appropriate definitions and details.) If $R$ is right nonsingular, then the class of all singular right $R$-modules forms the torsion class of a torsion theory which is usually known as the Goldie theory. A right nonsingular ring $R$ is said to have the splitting property (SP), if $Z(M)$ is a direct summand of $M$ for every right $R$-module $M$. A ring $R$ is said to have the finitely generated splitting property (FGSP), if $Z(M)$ is a direct summand of $M$ for every finitely generated right $R$-module $M$.

If $R$ is a ring, the Jacobson radical of $R$ will be denoted by $J(R)$. The prime radical of a ring $R$ is denoted by $N(R)$. A ring is semiprimary if $R / J(R)$ is semisimple Artinian and $J(R)$ is nilpotent. In this case, $N(R)=J(R)$.

A nonzero element of a ring $R$ is a left (right) regular element if it has zero left (right) annihilator. A regular element is a left and right regular element. A ring $Q$ is said to be a classical right quotient ring of $R$ ( $R$ is called a right order in $Q$ ) if (i) $R \subseteq Q$, (ii) every element of $Q$ has the form $a c^{-1}$ when $a, c \in R$ with $c$ a regular element in $R$, and (iii) every regular element of $R$ has an inverse in $Q$. Classical left quotient rings are defined similarly.

An $R$-module $M$ is almost finitely generated (AFG) if $M / \operatorname{soc}(M)$ is finitely generated. An $R$-module $M$ is almost finitely related (AFR) if there exists an exact sequence of $R$-modules

$$
0 \longrightarrow K \longrightarrow F \longrightarrow M \longrightarrow 0
$$

where $F$ is a finitely generated free $R$-module and $K$ is AFG. We refer the reader to [11] or [16] for further details. 
Formal triangular matrix rings will play a central role in this paper. For a more detailed discussion of such rings see [15] or [17]. The following proposition is due to Goodearl [17, Proposition 3 and Proposition 4] or [15, Propositions 4.2 and 4.4].

Proposition 1.1. Let $R=\left[\begin{array}{cc}A & 0 \\ B & C\end{array}\right]$ where $B$ is a $C-A$ bimodule and ${ }_{\sigma} B$ is faithful. Then the following statements hold:

(a) $A$ right ideal $I$ of $R$ belongs to $\mathscr{S}(R)$ if and only if it contains a right ideal of the form $\left[\begin{array}{ll}J & 0 \\ K & 0\end{array}\right]$ where $J \in \mathscr{S}(A)$ and $K_{A}$ is essential in $B_{\Delta}$.

(b) $R_{R}$ is nonsingular if and only if $A_{A}$ and $B_{A}$ are both nonsingular.

(c) $\operatorname{soc}\left(R_{R}\right)=\left[\begin{array}{ll}\operatorname{soc}\left(A_{A}\right) & 0 \\ \operatorname{soc}\left(B_{A}\right) & 0\end{array}\right]$.

(d) If $R_{R}$ is nonsingular, then $S^{0} R=\left[\begin{array}{ll}S^{0} A & X \\ S^{0} B & T\end{array}\right]$ where $X=\operatorname{Hom}_{A}\left(S^{0} B\right.$, $\left.S^{0} A\right)$ and $C$ is a unital subring of $T=\operatorname{End}_{A}\left(S^{0} B\right)$.

2. Rings with essential socle and FGSP. In this section a characterization is given of right nonsingular rings $R$ with essential right socle having FGSP which either are right finite dimensional or possess a semiprimary classical right quotient ring. We will use the methods of [8] to give a matrix representation of such rings which will be quite explicit if $R$ is right finite dimensional and has a semiprimary classical right quotient ring.

The following result due to Goodearl [16, Theorem 4.9] specialized to the case when $R$ has essential right socle is the starting point of our investigation.

THEOREM 2.1. Let $R$ be a right nonsingular ring with essential right socle. Then $R$ has FGSP if and only if

(a) $\{r \in R: x r \in A\}$ is $A F G$ for any $x \in S^{\circ} R$ and any finitely generated submodule $A$ of $\left(S^{\circ} R\right)_{R}$.

(b) If $I$ is any left ideal of $R$ containing $\operatorname{soc}\left(R_{R}\right)$, then $I$ is flat and $\operatorname{Tor}_{1}{ }^{R}\left(S^{0} R, R / I\right)=0$.

If $R$ is right nonsingular with essential right socle which either is right finite dimensional or possesses a semiprimary classical right quotient ring, then $\operatorname{soc}\left(R_{R}\right)=R e$ for $e^{2}=e$ by Gordon [20, Theorem 1.2]. Let $f=1-e$. Then $R$ is isomorphic to $\left[\begin{array}{cc}e R e & 0 \\ f R e & f R f\end{array}\right]=\left[\begin{array}{ll}A & 0 \\ B & C\end{array}\right]$ where $A$ a semisimple Artinian, ${ }_{C} B_{A}$ is a $C-A$-bimodule faithful as a $C$-module. By Proposition $1.1 S^{0} R$ is isomorphic to $\left[\begin{array}{ll}A & X \\ B & T\end{array}\right]$ where $X=\operatorname{Hom}_{A}(B, A)$ and $T=\operatorname{End}_{A}(B)=f\left(S^{0} R\right) f$. We will use 
this notation throughout this section.

We proceed with a sequence of lemmas, the first of which is [8, Lemma 3.4] included here for completeness.

Lemma 2.2. $\operatorname{Tor}_{1}{ }^{R}\left(S^{0} R, R / I\right)=0$ for all left ideals $I$ of $R$ contained in $C=R f$ if and only if $T$ is a right $C$-flat overring of C.

Lemma 2.3. Let $S$ be a semihereditary ring and suppose that $S$ is embedded in a left finite dimensional ring $U$ such that $U$ is right $S$-flat. Then $S$ possesses a left Artinian classical left quotient ring.

Proof. By Gordon and Small [21], it is sufficient to show that $S$ is left finite dimensional. Since $U_{S}$ is $S$-flat, $U(L \cap K)=U L \cap U K$ for left ideals $K$ and $L$ of $S$ by [3, Lemma 1.10]. From this it follows that if $I_{1} \oplus \cdots \oplus I_{n}$ is a direct sum of left ideals of $S$, then $U I_{1} \oplus \cdots \oplus U I_{n}$ is a direct sum of left ideals of $U$. Since $U$ is left finite dimensional, then $S$ is left finite dimensional.

REMARK. Semihereditary rings with semiprimary classical left quotient rings are characterized by [10, Theorem 3.7].

Using the notation that has been prescribed, we have the following lemma.

Lemma 2.4. If $T$ is right $C$-flat and $C$ possesses a classical left quotient ring $Q^{\prime}$, then $Q^{\prime} \leqq T$.

Proof. First we show that a regular element $d$ of $C$ is a regular element of $T$. Since $T$ is semisimple Artinian, it suffices to show that the left annihilator of $d$ in $T$ is zero. For let $t d=0$ for $t \in T$. Since $T$ is right $C$-flat, by [2, Problem 5, p. 122] there exists $\left\{t_{j}\right\} \subseteq T,\left\{\lambda_{j}\right\} \subseteq C$ such that $t=\sum t_{j} \lambda_{j}$ and $\lambda_{j} d=0$ for all $j$. Since $d$ is a regular element of $C, \lambda_{j}=0$ for all $j$ and $t=0$.

Thus every regular element of $C$ is invertible in $T$ since $T$ is semisimple Artinian. Hence $C \subseteq Q^{\prime} \subseteq T$.

Now we state the first main result of this section.

THEOREM 2.5. Let $R$ be a right nonsingular right finite dimensional ring which has an essential right socle and FGSP. Then $R$ is isomorphic to $\left[\begin{array}{ll}A & 0 \\ B & C\end{array}\right]$ where

(i) $A$ is a semisimple Artinian ring; 
(ii) $B$ is a $C-A$ bimodule finitely generated as an $A$ module;

(iii) $C$ is a semihereditary ring possessing a left Artinian classical left quotient ring $Q^{\prime} \subseteq T=\operatorname{End}_{A}(B)$ such that $T$ is a right flat $Q^{\prime}$-module.

Conversely, any such ring is a right nonsingular right finite dimensional ring which has an essential right socle and FGSP.

Proof. Let $R$ satisfy the hypothesis of the theorem. By the remarks following Theorem 2.1 we may assume that $R$ is isomorphic to $\left[\begin{array}{cc}A & 0 \\ B & C\end{array}\right]=\left[\begin{array}{cc}e R e & 0 \\ f R e & f R f\end{array}\right]$ where $A$ is semisimple Artinian, $B$ is a $C-A$ bimodule and $C \subseteq T=\operatorname{End}_{A}(B)$. Since $R$ is right finite dimensional, $B$ is finitely generated as a right $A$-module.

Now we show that $C$ is semihereditary and $T$ is right $C$-flat. Let $I$ be a left ideal of $C$. Then $I$ is a left ideal of $R$ contained in $R f$; so by Theorem 2.1, $R e \oplus I$ is a flat left ideal of $R$. Hence $I$ is $R$-flat and by [9; Proposition 2.1] $I$ is $C$-flat. Since $R_{R}$ is finite dimensional, $T=\operatorname{End}_{A}(B)$ is semisimple Artinian. As $C$ is a unital subring of $T$, by [23, Corollary 3.2] any finitely generated $C$-flat module is $C$-projective. It then follows that $C$ is left semihereditary; hence by Small [28, Theorem 3] $C$ is semihereditary. By Theorem 2.1 (b) we have that $\operatorname{Tor}_{1}{ }^{R}\left(S^{0} R, R /(R e \oplus I)\right)=0$ for all left ideals $I$ of $C$. Since $R=R e \oplus R f, R / I \cong R e \oplus R f / I$; but $R f / I \cong$ $R /(R e \oplus I)$. It follows that $\operatorname{Tor}_{1}{ }^{R}\left(S^{0} R, R / I\right)=0$ since $R \mathrm{e}$ is left $R$-projective. By Lemma 2.2, $T$ is right $C$-flat.

By Lemma 2.3, $C$ has a left Artinian classical left quotient ring $Q^{\prime}$ and by Lemma $2.4 Q^{\prime} \cong T$. Since $Q^{\prime}$ is a classical left quotient ring of $C, T$ is right $Q^{\prime}$-flat by [30, Proposition 3.11, p. 232].

Conversely, let $R$ be as in the conclusion of the theorem. It follows from the triangular structure of $R$ that $R$ is a right nonsingular right finite dimensional ring with essential right socle. In order to show that $R$ has FGSP we will show that $R$ satisfies conditions (a) and (b) of Theorem 2.1. To see (b) let $I$ be a left ideal of $R$ containing $\operatorname{soc}\left(R_{R}\right)=R e$. Then $I=R e \oplus I f$. Thus If is a left ideal of $C$; so If is $C$-flat since $C$ is semihereditary. Therefore If is $R$-flat by [9, Proposition 2.1] and $I$ is $R$-flat. Since $Q^{\prime}$ is a classical left quotient ring of $C$ and $T_{Q^{\prime}}$ is $Q^{\prime}$-flat, it follows that $T_{C}$ is $C$-flat. By Lemma 2.2, $\operatorname{Tor}_{1}^{R}\left(S^{0} R, R / I f\right)=0$ and it follows that $\operatorname{Tor}_{1}^{R}\left(S^{0} R, R / I\right)=0$. Thus (b) holds.

To establish (a), by [16, Proposition 4.8] it is equivalent to show that all finitely generated submodules $M$ of $\left(S^{0} R\right)_{R}$ are AFR. Furthermore, by the discussion of $[16$, p. 65] it is sufficient to show that $M / M e$ is $C$-finitely related. Now 


$$
S^{0} R=\left[\begin{array}{ll}
e\left(S^{0} R\right) e & e\left(S^{0} R\right) f \\
f\left(S^{0} R\right) e & f\left(S^{0} R\right) f
\end{array}\right]
$$

and $e\left(S^{0} R\right) f$ is isomorphic to a direct sum of direct summands of $T=f\left(S^{0} R\right) f$ as a right $T$-module. Since $T$ is right $C$-flat, then $e\left(S^{0} R\right) f$ is right $C$-flat; hence $\left(S^{0} R\right) f$ is a right $C$-flat module. $M / M e$ is a finitely generated submodule of $\left(S^{0} R\right) f$ and hence is $C$-flat as $C$ is semihereditary. Therefore $M / M e$ is $C$-projective since finitely generated flat $C$-modules are $C$-projective by [23, Corollary 3.2]. Thus $M / M e$ is finitely related as a $C$-module; so $M$ is AFR.

REMARK. If $R=\left[\begin{array}{ll}A & 0 \\ B & C\end{array}\right]$ satisfies the conditions of the theorem, then $B$ is a left $Q^{\prime}$-module as $Q^{\prime} \cong T=\operatorname{End}_{A}(B)$. Hence $\left[\begin{array}{cc}A & 0 \\ B & Q^{\prime}\end{array}\right]$ is a semiprimary ring with $T_{Q^{\prime}}$ right $Q^{\prime}$-flat. Thus by [8, Theorem 3.5], $\left[\begin{array}{rr}A & 0 \\ B & Q^{\prime}\end{array}\right]$ is a splitting ring.

We now turn our attention to right nonsingular rings with essential right socle which possess a semiprimary classical right quotient ring. As we have observed earlier, $R=\left[\begin{array}{cc}A & 0 \\ B & C\end{array}\right]=\left[\begin{array}{cc}e R e & 0 \\ f R e & f R f\end{array}\right]$ where $A$ is a semisimple Artinian ring and ${ }_{C} B_{A}$ is a bimodule faithful as a $C$-module. In addition, finitely generated flat $R(C)$-modules are $R(C)$-projective by [23, Corollary 3.2]. A result similar to Theorem 2.5 holds in this case; however, we first need the following lemma which will be useful later in this paper.

LEMMA 2.6. Let $R$ be a right nonsingular ring isomorphic to

$$
\left[\begin{array}{ll}
A & 0 \\
B & C
\end{array}\right]
$$

where $A$ is a right finite dimensional semiprime ring, $B$ is a $C-A$ bimodule faithful xs a left $C$-module and $C$ has a semiprimary classical right quotient ring $Q^{\prime}$ such that $Q^{\prime} \subseteq T=\operatorname{End}_{A}\left(S^{0} B\right)$. Then $R$ has a semiprimary classical right quotient ring $Q$ where

$$
Q=\left[\begin{array}{cc}
S^{0} A & 0 \\
S^{0} B & Q^{\prime}
\end{array}\right]
$$

Proof. The hypothesis implies that $Q$ is a semiprimary ring. It remains to show that $Q$ is a classical right quotient ring of $R$. Let $d=\left[\begin{array}{ll}a & 0 \\ b & c\end{array}\right]$ be a regular element of $R$ where $a \in A, b \in B$, and $c \in C$. Then $a$ and $c$ are regular elements of $A$ and $C$ respectively; so they have inverses $x$ and $y$ in $S^{0} A$ and $Q^{\prime}$ respectively as $S^{0} A$ 
and $Q^{\prime}$ are classical right quotient rings of $A$ and $C$. Since $S^{\circ} B$ is a left $Q^{\prime}$-module and $c$ is invertible in $Q^{\prime}, c S^{0} B=S^{0} B$. Hence there exists $w \in S^{0} B$ such that $c w+b x=0$. Then

$$
\left[\begin{array}{ll}
a & 0 \\
b & c
\end{array}\right]\left[\begin{array}{ll}
x & 0 \\
w & y
\end{array}\right]=\left[\begin{array}{ll}
1 & 0 \\
0 & 1
\end{array}\right]
$$

and it follows that $d$ is invertible in $Q$.

Let $x=\left[\begin{array}{ll}a & 0 \\ b & c\end{array}\right] \in Q$ where $a \in S^{0} A, b \in S^{0} B$, and $c \in Q^{\prime}$. Since $B\left(S^{0} A\right)=S^{0} B$ and since $A$ and $C$ have classical right quotient rings $S^{0} A$ and $Q^{\prime}$ respectively, $a=a_{1} d_{1}^{-1}, b=b_{1} d_{2}^{-1}$, and $c=c_{1} d_{3}^{-1}$ where $a_{1} \in A, b_{1} \in B, c_{1} \in C, d_{1}$ and $d_{2}$ are regular elements in $A$ while $d_{3}$ is a regular element of $C$. Let $d$ be a common left multiple of $d_{1}^{-1}$ and $d_{2}^{-1}$; i.e., $d_{1}^{-1} d$ and $d_{2}^{-1} d \in A$. Then

$$
\left[\begin{array}{ll}
a & 0 \\
b & c
\end{array}\right]\left[\begin{array}{cc}
d & 0 \\
0 & d_{3}
\end{array}\right] \in R
$$

Since $\bar{d}=\left[\begin{array}{cc}d & 0 \\ 0 & d_{3}\end{array}\right]$ is a regular element in $R, \lambda=r \bar{d}^{-1}$ for $\bar{d}$ a regular element of $R$ and some $r \in R$. Hence $Q$ is the classical right quotient ring of $R$.

We have the following analogue to Theorem 2.5.

Proposition 2.7. Let $R$ be a right nonsingular ring with essential right socle which possesses a semiprimary classical right quotient ring $Q$ and FGSP. Then $R$ is isomorphic to $\left[\begin{array}{ll}A & 0 \\ B & C\end{array}\right]$ where

(i) $A$ is a semisimple Artinian ring;

(ii) $B$ is a $C-A$ bimodule;

(iii) $C$ is a semihereditary ring possessing a semiprimary classical right quotient ring $Q^{\prime} \subseteq T=\operatorname{End}_{A}(B)$ and $T$ is a flat right C-module.

Conversely any such ring $R$ is a right nonsingular ring with essential right scole which possesses a semiprimary classical right quotient ring and FGSP.

Proof. Let $R$ satisfy the hypothesis of the theorem. Then the techniques used in proving Theorem 2.5 can be used to show $R$ satisfies all of (i)-(iii) except that $C$ possesses a semiprimary classical right quotient ring $Q^{\prime} \subseteq T$. But this follows from the fact that $C=f R f, Q \subseteq S^{\circ} R$ and $f Q f$ is a semiprimary classical right quotient ring of $C$ by [18, Theorem A]. 
For the converse, again the techniques of Theorem 2.5 apply to show that $R$ is a right nonsingular ring with essential right socle and FGSP. By Lemma 2.6, $R$ possesses a semiprimary classical right quotient ring.

Using the fact that a ring which possesses a classical left quotient ring and a classical right quotient ring possesses a classical two-sided quotient ring, Theorem 2.5 and Proposition 2.7 combine to give the following.

THEOREM 2.8. Let $R$ be a right nonsingular right finite dimensional ring with essential right socle and FGSP which possesses a semiprimary classical right quotient ring. Then $R$ is isomorphic to $\left[\begin{array}{ll}A & 0 \\ B & C\end{array}\right]$ where

(i) $A$ is a semisimple Artinian ring;

(ii) $B$ is a $C-A$ bimodule finitely generated as an $A$-module;

(iii) $C$ is a semihereditary ring possessing a left Artinian two-sided classical quotient ring $Q^{\prime} \leqq T=\operatorname{End}_{A}(B)$ such that $T$ is a right $Q^{\prime}$-flat.

Conversely any such ring $R$ is a right nonsingular right finite dimensional ring with essential right socle and FGSP which possesses a semiprimary classical right quotient ring.

Suppose that $R$ satisfies either Theorem 2.5 or Theorem 2.8 and has homogeneous right socle. Then $S^{0} R$ is a finite dimensional matrix ring over a division ring and it follows that $T$ is also. Now $Q^{\prime}$ is a hereditary ring since $C$ is semihereditary; also $T$ is right $Q^{\prime}$-flat. Hence by [10, Theorem 2.1] $Q^{\prime}$ has a very explicit structure in terms of matrix rings over division rings which in turn induces an explicit structure on $C$ by [10, Theorem 3.7 and Theorem 3.11]. Thus a quite explicit representation can be obtained if $R$ has homogeneous socle. Now we will give an explicit subdirect representation of $R$ in terms of rings with homogeneous socle.

If $R$ is the subdirect product of $R_{1}$ and $R_{2}$, we define $E_{1}$ and $E_{2}$ by the relations $E_{1} \times 0=R \cap\left(R_{1} \times 0\right)$ and $0 \times E_{2}=R \cap\left(0 \times R_{2}\right)$. $E_{1}$ and $E_{2}$ are two-sided ideals of $R_{1}$ and $R_{2}$ respectively and $R$ is said to be a (right) split product of $R_{1}$ and $R_{2}$ if $E_{1}$ and $E_{2}$ are direct summands of $R_{1}$ and $R_{2}$ respectively as right ideals. For further details we refer the reader to [13].

Let $R$ be a right finite dimensional right nonsingular ring with essential right socle. Then there exists orthogonal idempotents $\left\{e_{1}, \cdots, e_{t}\right\}$ such that $R e_{1} \oplus \cdots \oplus R e_{t}=\operatorname{soc}\left(R_{R}\right)$ and $R e_{i}$ is homogeneous for $i=1, \cdots, t$. By Gordon [19, Theorem 2.2] $R$ is a unique subdirect product of rings $R_{1}, \cdots, R_{t}$ such that $R_{i}$ has essential 
homogeneous right socle isomorphic to $R \mathrm{e}_{i}$ for $i=1, \cdots, t$. The minimal right ideals of $R_{i}$ are $R$-isomorphic to the minimal right ideals of $R$ by $[19, \mathrm{p} .312]$; hence each $R_{i}$ is a right nonsingular right $R$-module. Now $S^{0} R \cong S^{0} R_{1} \times \cdots \times S^{0} R_{t}$ (ring direct product). For any $X \subset\{1, \cdots, t\}$ let $\pi$ denote the projection map of $S^{0} R$ onto $\mathrm{X}_{i \in X} S^{0} R_{i}$. Let $R_{X}=\pi_{X}(R), e=e_{1}+\cdots+e_{t}$ and $f=1-e$. Let $e_{X}=\pi_{X}(e)$ and $f_{X}=\pi_{X}(f)$. Letting $T$ and $C$ be as in Theorem 2.5, set $T_{X}=f_{X} T f_{X}$ and $C_{X}=f_{X} R f_{X}$. For a partition $X$ and $Y$ of $\{1, \cdots, t\}, R$ is a subdirect product of $R_{X}$ and $R_{Y}$ while $C$ is a subdirect product of $C_{X}$ and $C_{Y}$. Let $E_{X}$ and $E_{Y}$ be the two-sided ideals of $R_{X}$ and $R_{Y}$ respectively by defining $E_{X} \times 0=\left(R_{X} \times 0\right) \cap R$ and $0 \times E_{Y}=\left(0 \times R_{Y}\right) \cap R$. Let $E_{X}^{\prime}=f_{X} E_{X} f_{X}$ and $E_{Y}^{\prime}=f_{Y} E_{Y} f_{Y}$. Using this notation, we have the following theorem.

THEOREM 2.9. Let $R$ be a right nonsingular right finite dimensional ring with essential right socle. Then the following statements are equivalent:

(i) $R$ has FGSP;

(ii) For any partition $X$ and $Y$ of $\{1, \cdots, t\}$

(a) $R_{X}$ and $R_{Y}$ are right nonsingular right finite dimensional rings with essential right socle and FGSP;

(b) $C$ is a right split product of $C_{X}$ and $C_{Y}$.

If, in addition, $R$ possesses a semiprimary classical right quotient ring, then $R$ has FGSP if and only if, in addition, $R_{X}$ and $R_{Y}$ both possess semiprimary classical right quotient rings.

Proof. (i) $\Rightarrow$ (ii): It follows from the construction of $R_{X}$ and $R_{Y}$ that $R_{X}$ and $R_{Y}$ are right nonsingular right finite dimensional rings with essential socle. It follows from what we have observed that the right socles of $R_{X}$ and $R_{Y}$ are $R$-nonsingular; so $R_{X}$ and $R_{Y}$ are $R$-nonsingular. Since $R_{X}$ and $R_{Y}$ are homomorphic images of $R, R_{X}$, and $R_{Y}$ have FGSP by [16, Proposition 1.11].

Since $S^{0} R=S^{0} R_{X} \times S^{0} R_{Y}$, it follows that $T=T_{X} \times T_{Y}$. Since $T$ is right $C$-flat and $C$ is semihereditary by Theorem 2.5 , then $C$ is a split product of $C_{X}$ and $C_{Y}$ respectively as right ideals by [9, Proposition 3.8].

(ii) $\Rightarrow$ (i): To show that $R$ has FGSP, it suffices to show that $C$ is semihereditary and $T_{C}$ is $C$-flat by Theorem 2.5. By (ii) (a) and Theorem 2.5, $C_{X}$ and $C_{Y}$ are semihereditary and $T_{X}$ and $T_{Y}$ are respectively right $C_{X}$ and $C_{Y}$-flat. Since $C$ is a split product of $C_{X}$ and $C_{Y}, E_{X}^{\prime}$ and $E_{Y}^{\prime}$ are direct summands of $C_{X}$ and $C_{Y}$ respectively as right ideals. By [9, Proposition 2.1] $T_{X}$ is right $C$-flat since $C_{X}=C /\left(0 \times E_{Y}^{\prime}\right)$ and $0 \times E_{Y}^{\prime}$ is a direct summand of $C$ as a right 
ideal. Similarly $T_{Y}$ is right $C$-flat. Hence $T=T_{X} \times T_{Y}$ is $C$-flat. By [9, Theorem 3.10] $C$ is semihereditary and $R$ has FGSP.

In order to show the last statement let, in addition, $R$ have a semiprimary classical right quotient ring $Q$ and FGSP. By Lemma 2.6 to show that $R_{X}$ and $R_{Y}$ have semiprimary classical right quotient rings it suffices to show that $C_{X}$ and $C_{Y}$ have semiprimary classical right quotient rings $Q_{X} \subseteq T_{X}$ and $Q_{Y} \subseteq T_{Y}$. By [18, Theorem A] $C$ has a semiprimary classical right quotient ring $Q^{\prime} \leqq T$. Since $C$ is a split product of $C_{X}$ and $C_{Y}$ by (ii), then $C_{X}$ and $C_{Y}$ have semiprimary classical right quotient rings $Q_{X}^{\prime}$ and $Q_{Y}^{\prime}$ where $Q^{\prime}$ is the split product of $Q_{X}^{\prime}$ and $Q_{Y}^{\prime}$ by [9, Theorem 3.17]. Hence it follows that $Q_{X}^{\prime} \subseteq T_{X}$ and $Q_{Y}^{\prime} \subseteq T_{Y}$ as desired.

Now assume that $R_{X}, R_{Y}, C_{X}, C_{Y}$, satisfy the conditions of the theorem and $R_{X}, R_{Y}$ have semiprimary classical right quotient rings $Q_{X}$ and $Q_{Y}$. By Theorem 2.8, $C_{X}$ and $C_{Y}$ have semiprimary classical right quotient rings $Q_{X}^{\prime} \subseteq T_{X}$ and $Q_{Y}^{\prime} \subseteq T_{Y}$ respectively. $C$ is a split product of $C_{X}$ and $C_{Y}$; so by [9, Theorem 3.17] $C$ has a semiprimary classical right quotient ring $Q^{\prime}$ which is a split product of $Q_{X}^{\prime}$ and $Q_{Y}^{\prime}$. Since $T=T_{X} \times T_{Y}, Q^{\prime} \subseteq T$. Hence $R$ has a semiprimary classical right quotient ring by Lemma 2.6.

We conclude this section with some examples. The first is an example which satisfies Theorem 2.7 but not Theorem 2.5. Let $R$ be the ring

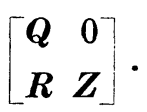

Where $\boldsymbol{Q}$ is the rational numbers, $\boldsymbol{R}$ is the real numbers and $\boldsymbol{Z}$ is the ring of integers. Then $R$ has semiprimary classical right quotient ring $\left[\begin{array}{ll}\boldsymbol{Q} & 0 \\ \boldsymbol{R} & \boldsymbol{Q}\end{array}\right]$ but is clearly not right finite dimensional. Note that $R$ has homogeneous right socle.

The second example is an example of a ring which satisfies the conditions of Theorem 2.5 but does not have a semiprimary classical right quotient ring. Let $F$ be a field and $x$ an indeterminate over $F$. As usual $F[x]$ is the ring of polynomials over $F$ and $F(x)$ the quotient field of $F[x]$. By [18, p. 42-43] the ring

$$
R=\left[\begin{array}{ccc}
F(x) & 0 & 0 \\
F(x) & F & 0 \\
F(x) & F[x] & F[x]
\end{array}\right)
$$

possesses a semiprimary classical left quotient ring but not a semiprimary classical right quotient ring. The ring $C=\left[\begin{array}{cc}F & 0 \\ F[x] & F[x]\end{array}\right]$ 
has a classical left quotient ring $Q^{\prime}=\left[\begin{array}{ll}F & 0 \\ F(x) & F(x)\end{array}\right]$ and $T=\left[\begin{array}{ll}F(x) & F(x) \\ F(x) & F(x)\end{array}\right]$ is right $Q^{\prime}$-flat. Hence $R$ satisfies the conditions of Theorem 2.5. Again $R$ has homogeneous socle.

3. Rings with zero socle and FGSP. In this section the structure of right nonsingular rings with zero socle and FGSP is examined. It is shown that if $R$ is a right nonsingular ring with zero socle and FGSP, then $R$ is right finite dimensional if and only if $R$ possesses a semiprimary classical right quotient ring. This is contrary to the situation in the previous section. Then the structure of right nonsingular right finite dimensional rings having zero socle and FGSP is given as a certain triangular matrix ring and reduces the problem of characterizing rings with zero socle and FGSP to studying semiprime rings. Finally these results are applied to certain classes of rings to obtain more explicit results. Many of the ideas of this section were motivated by the ideas of Goodearl's paper [17].

We begin by studying the structure of right nonsingular rings $R$ which have FGSP, zero socle and a semiprimary classical right quotient ring. We need the follwing technical lemma which is a slight modification of [17, Lemma C].

LEMMA 3.1. Let $R$ have zero socle and FGSP, and let $M$ be a two-sided ideal in $L^{*}(R)$ whose left annihilator $H$ is an essential right ideal of $R$. Then $M$ is a direct summand. of $R$ as a right ideal.

Proof. Since $M /(M \cap H) \cong(M+H) / H$ is right singular and $R / M$ is right nonsingular, by FGSP the exact sequence

$$
0 \longrightarrow M /(M \cap H) \longrightarrow R /(M \cap H) \longrightarrow R / M \longrightarrow 0
$$

splits. Then there exists an $m \in M$ such that $m R+M \cap H=M$ and $m^{2}-m \in M \cap H$. Then it follows that $m^{3}-m \in M \cap H$; so that $m^{4}-m^{2}=0$. Hence $m^{2}=e$ is an idempotent of $R$. Now $e R+M \cap$ $H=M+M \cap H=M$. Therefore $e M=M^{2}$ multiplying the previous equation on the right by $M$. Hence $e R=M^{2}$ and $M^{2}$ is a direct summand of $R_{R}$.

If $M^{2} \neq M$, by [17, Lemma B] $M_{R}$ must have a proper essential submodule $K$ which contains $M^{2}$. It follows that $M / K=Z(R / K)$ and $M / K$ is a direct summand of $R / K$. Thus there is a right ideal $J$ of $R$ such that $M+J=R$ and $M \cap J=K$. Since $M=M^{2}+J M$, we have that $M \cong J$. But this implies that $M=K$, a contradiction. 
Let $R$ be a right nonsingular ring with zero socle having FGSP and suppose that $R$ possesses a semiprimary classical right quotient ring $Q$. Then $Q$ is a right nonsingular ring and $\operatorname{soc}\left(Q_{Q}\right)=$ $Q e$ for an idempotent $e$ of $Q$. If $f=1-e$, then $Q$ is isomorphic to

$$
\left[\begin{array}{cc}
e Q e & 0 \\
f Q e & f Q f
\end{array}\right] \text {. }
$$

Let $M=R \cap f Q . \quad f Q$ is a two-sided ideal of $Q$; so $M$ is a twosided ideal of $R$. $Q e \subseteq l_{Q}(f Q)$; so $R \cap Q e \subseteq l_{R}(M)$. Hence $H=l_{R}(M)$ is essential as a right ideal of $R$. Furthermore $R / M \cong R /(R \cap f Q) \cong$ $(R+f Q) / f Q$; so $R / M$ is a right nonsingular $R$-module. Hence by Lemma 3.1, $M=f^{\prime} R$ for $f^{\prime}$ an idempotent element of $R$. Since $M_{R}$ is essential in $f Q$, it follows that $f Q=f^{\prime} Q$. Let $e^{\prime}=1-f^{\prime}$. Now $Q=e Q \oplus f Q$; so $e Q=e^{\prime} Q$. Since $e^{\prime} Q f^{\prime} \cong e^{\prime} Q f^{\prime} Q=e Q f Q=0, Q=$ $\left[\begin{array}{cc}e^{\prime} Q e^{\prime} & 0 \\ f^{\prime} Q e^{\prime} & f^{\prime} Q f^{\prime}\end{array}\right]$. Since $e Q=e^{\prime} Q$, then $e^{\prime} Q e^{\prime}=e Q e$. Since $f Q e=$ $(f Q)(e Q e)=\left(f^{\prime} Q\right)\left(e^{\prime} Q e^{\prime}\right)=f^{\prime} Q e^{\prime}$, then $Q e=e Q e+f Q e=e^{\prime} Q e^{\prime}+f^{\prime} Q e^{\prime}=$ $Q e^{\prime}$. It follows that we can take the idempotents $e$ and $f$ of $Q$ to be idempotent elements of $R$ as well and that

$$
R=\left[\begin{array}{cc}
e R e & 0 \\
f R e & f R f
\end{array}\right]=\left[\begin{array}{ll}
A & 0 \\
B & C
\end{array}\right] \text {. }
$$

Since $f Q e$ is a faithful $f Q f$-module, it follows that $B$ is a faithful left $C$-module. Hence by Proposition 1.1 we have

$$
S^{0} R=\left[\begin{array}{cc}
S^{0} A & X \\
S^{0} B & T
\end{array}\right]
$$

where $T=\operatorname{End}_{A}\left(S^{0} B\right)$ and $C$ is a unital subring of $T$. Since $Q$ is the classical right quotient ring of $R$, we may assume that $R \subseteq Q \subseteq$ $S^{0} R$ and

$$
Q \cong\left[\begin{array}{cc}
S^{0} A & 0 \\
S^{0} B & Q^{\prime}
\end{array}\right]
$$

where $Q^{\prime}=f Q f \leqq T$. We will assume that $R, Q, f, e$ and $S^{\circ} R$ are as described and that $R$ has FGSP for Lemmas 3.2 through 3.4.

Applying the proof of [17, Lemma D] to the idempotent $f$ and noting that the proof of [17, Lemma D] only uses FGSP, we have the following.

Lemma 3.2. $S^{0} C=T$.

LEMMA 3.3. $C$ is semihereditary and $T$ is the two-sided maximal quotient ring of $C$. 
Proof. $C$ has a semiprimary classical right quotient ring by [18, Theorem A] and hence finitely generated flat left and right $C$-modules are $C$-projective. Since $R e$ is essential in $R_{R}$, it follows from [16, Proposition 4.2] that $C$ is semihereditary. It also follows from Lemma 2.2 that $T$ is right $C$-flat. Since $T$ is the maximal right quotient ring of $C$ by Lemma 3.2, every finitely generated nonsingular right $C$-module can be embedded in a finite direct sum of copies of $T$. Hence any finitely generated nonsingular right $C$ module is $C$-projective. Therefore by [4, Theorem 2.3] $Z\left(T \otimes \otimes_{C} T\right)=$ 0 ; so $T \otimes{ }_{C} T \cong T$. Thus by [5, Proposition 2.2], $T$ is a left quotient ring of $C$.

Let $U$ be the maximal left quotient ring of $C$ chosen so that $C \subseteq T \subseteq U$. Then $U$ is a left flat $C$-module by [3, Theorem 2.1]; so again by the argument used in the previous paragraph, every finitely generated nonsingular left $C$-module is $C$-projective. Thus by [4, Theorem 2.3] $Z\left(U \otimes{ }_{c} U\right)=0$ and by [5, Proposition 2.2] $U$ is a right quotient ring of $C$. Therefore it follows that $U \subseteq T$ or $U=T$.

Lemma 3.4. $T$ is semisimple Artinian and $C$ has a two-sided Artinian classical two-sided quotient ring $Q^{\prime}$.

Proof. Since $S^{0} R$ is a direct product of full linear rings being the maximal quotient ring of the semiprimary ring $Q, T$ is the direct product of full linear rings as well. By Lemma 3.3, $T$ is two-sided self-injective and $T$ is semisimple Artinian by Osofsky [24]. Hence the classical right quotient ring $Q^{\prime}$ of $C$ exists and is a two-sided classical quotient ring as well as two-sided Artinian by Lemma 2.3.

REMARK. Theorem 3.14 of [10] gives a complete characterization of $C$ and $Q^{\prime}$.

We now show that for right nonsingular rings $R$ with zero socle and FGSP, $R_{R}$ is finite dimensional if and only if $R$ possesses a semiprimary classical right quotient ring.

Proposition 3.5. Let $R$ be a right nonsingular ring with zero socle and FGSP. Then $R_{R}$ is finite dimensional if and only if $R$ possesses a semiprimary classical right quotient ring.

Proof. Suppose that $R$ has a semiprimary classical right quotient ring $Q$. Then we know that $R=\left[\begin{array}{cc}e R e & 0 \\ f R e & f R f\end{array}\right]=\left[\begin{array}{cc}A & 0 \\ B & C\end{array}\right]$ where 
$Q e=\operatorname{soc}\left(Q_{Q}\right)$. Since $Q$ is semiprimary, $e Q e=S^{0} A$ is semisimple Artinian. Now $T$ is semisimple Artinian by Lemma 3.4; so it follows that $S^{0} R$ is semisimple Artinian. Consequently $R_{R}$ is finite dimensional by [26, Theorem 1.6].

Conversely let $R_{R}$ be finite dimensional. Let.$/$ be the set of two-sided ideals in $L^{*}(R)$ whose left annihilator is essential as a right ideal. Since $R_{R}$ is finite dimensional, $L^{*}(R)$ has ACC; hence $\therefore$ has a maximal element, say $M . R / M$ is a semiprime ring by the proof of Theorem 2 in [17].

By Lemma 3.1, $M=f R$ for an idempotent $f$. Set $e=1-f$. Then $R e$ is the left annihilator of $M$ so $R$ is isomorphic to $\left[\begin{array}{cc}e R e & 0 \\ f R e & f R f\end{array}\right]=$ $\left[\begin{array}{ll}A & 0 \\ B & C\end{array}\right]$ where $A$ is semiprime and $B$ is faithful. Thus by Proposition 1.1, $C$ is a unital subring of $T=\operatorname{End}_{A}\left(S^{0} B\right)$. The proofs of Lemmas 3.3 and 3.4 still apply to show that $T$ is the two-sided maximal quotient ring of the semihereditary ring $C$ and $T$ is semisimple Artinian. Hence by Lemma 2.3, $C$ has a two-sided Artinian two-sided classical quotient ring $Q^{\prime}$. Therefore $R$ possesses a semiprimary classical right quotient ring by Lemma 2.5 .

REMARK. We have actually shown that the classical right quotient ring of $R$ is right Artinian.

If $R=\left[\begin{array}{cc}A & 0 \\ B & C\end{array}\right]$ is a formal triangular matrix ring, for convenience we label the following two-sided ideals of $R$ :

$$
R_{12}=\left[\begin{array}{ll}
A & 0 \\
B & 0
\end{array}\right] \text { and } R_{23}=\left[\begin{array}{ll}
0 & 0 \\
B & C
\end{array}\right]
$$

The following theorem is the main result of this section.

THEOREM 3.6. Let $R$ be a right nonsingular right finite dimensional ring with zero socle and FGSP. Then $R$ is isomorphic to

$$
\left[\begin{array}{ll}
A & 0 \\
B & C
\end{array}\right]
$$

where

(i) A is a right nonsingular right finite dimensional semiprime ring with zero socle and FGSP;

(ii) $B$ is a $C-A$ bimodule such that $B_{A}$ is nonsingular and finite dimensional;

(iii) $C$ is a semihereditary ring possessing a two-sided Artinian 
two-sided classical quotient ring $Q^{\prime}$ such that $Q^{\prime} \subseteq T=\operatorname{End}_{A}\left(S^{\circ} B\right)$ and $T$ is the two-sided maximal quotient ring of $C$;

(iv) $\operatorname{Ext}_{A}^{1}\left(Z\left(N / N R_{23}\right), W\right)=0$ where $N$ is any finitely generated nonsingular right $R$-module and $W$ is any singular $A$-module.

Conversely, any such matrix ring is a right nonsingular right finite dimensional ring with zero socle and PGSP.

Proof. Let $R$ have FGSP. Since $A=R / R_{23}, A_{R}$ is right nonsingular. Hence the singular submodule of any right $A$-module is the same whether considered as an $A$-module or an $R$-module. Hence it follows that $A$ has FGSP. The remainder of the conditions of (i)-(ii) follow from Proposition 1.1, Lemma 3.1 and the fact that $R_{R}$ is finite dimensional. Condition (iii) is a consequence of Lemmas 3.3 and 3.4 .

To see condition (iv), let $N$ be any finitely generated right nonsingular $R$-module and $W$ a singular $A$-module. Then $W$ is $R$ singular; so by FGSP the exact sequence

$$
0 \longrightarrow W \longrightarrow X \longrightarrow N \longrightarrow 0
$$

splits. Since tensoring preserves direct sums, we have the following split exact sequence of $A$-modules,

$$
0 \longrightarrow W \longrightarrow X / X R_{23} \longrightarrow N / N R_{23} \longrightarrow 0 \text {. }
$$

Since $A$ has FGSP, $N / N R_{23}=Z\left(N / N R_{23}\right) \oplus U$. Hence we have $0 \cong$ $\operatorname{Ext}_{A}^{1}\left(N / N R_{23}, W\right) \cong \operatorname{Ext}_{A}^{1}\left(Z\left(N / N R_{23}\right), W\right)$.

The proof of the converse will require a sequence of lemmas. For Lemmas 3.7 through 3.10 , we will assume that $R=\left[\begin{array}{ll}A & 0 \\ B & C\end{array}\right]$ which satisfies (i)-(iv) of Theorem 3.6.

Lemma 3.7. ${ }_{C} B$ is flat and ${ }_{R} R_{23}$ is flat.

Proof. First we will show that ${ }_{C} B$ is nonsingular. $S^{0} B$ is a left $T$-module and since $T$ is semisimple Artinian, $S^{\circ} B$ is $T$-nonsingular. $T$ is the maximal left quotient ring of $C$; so $S^{0} B$ is $C$-nonsingular as well. Hence ${ }_{C} B$ is nonsingular.

Since $T$ is the two-sided maximal quotient ring of $C$, any finitely generated nonsingular left $C$-module can be embedded into a finite direct sum of copies of $T$. Since $C$ is semihereditary, by [3, Theorem 2.1] ${ }_{C} T$ is flat. Therefore any finitely generated nonsingular left $C$-module is flat. By a direct limit argument, any nonsingular left $C$-module is flat; hence ${ }_{C} B$ is flat.

Since ${ }_{C} B={ }_{R} B, B$ is a flat $R$-module by [9, Proposition 2.1]. $C$ 
is a direct summand of ${ }_{R} R$; so ${ }_{R} C$ is flat. As left $R$-modules, $R_{23}=$ $B \oplus C$; so $R_{23}$ is left $R$-flat.

The next lemma is motivated by [17, Lemma S].

Lemma 3.8. Let $K \in L^{*}\left(B^{n}\right)$ and $J=\left\{x \in C^{n}: x B \subseteq K\right\}$. Then $J B=K$ where $n$ is a positive integer.

Proof. Let $L=\left\{x \in T^{n}: x\left(S^{0} B\right) \subseteq S^{0} K\right\}$. Any element of $J$ or $L$ can be identified with an endomorphism of $S^{0} B^{n}$. Using this identification, we will show that $J \subseteq L$. Let $x \in J$. Since $x$ maps $B^{n}$ into $K, x$ induces a homomorphism $\bar{x}: S^{0} B^{n} / B^{n} \rightarrow S^{0} B^{n} / K$. Now $S^{0} B^{n}=S^{0} K \oplus$ $M$; so $S^{0} B^{n} / K \cong S^{0} K / K \oplus M$. Since $S^{0} B^{n} / B^{n}$ is a singular module, $\bar{x}\left(S^{0} B^{n} / B^{n}\right) \leqq S^{0} K / K$. Hence $x\left(S^{0} B^{n}\right) \leqq S^{0} K$ and $x \in L$.

Thus the natural mapping $\eta: C^{n} / J \rightarrow T^{n} / L$ is a well defined homomorphism. $J$ and $L$ are right $C$-modules, and we claim that $\eta$ is a monomorphism of right $C$-modules. For let $\eta(x+J)=0$. Then $x\left(S^{0} B\right) \subseteq S^{0} K$. But $x B \cong B^{n}$; so $x B \cong B^{n} \cap S^{0} K$. Now $K \cong B^{n} \cap$ $S^{0} K$ and $B^{n} \cap S^{0} K$ is an essential extension of $K$. But $K$ is closed in $B^{n}$; so $K=B^{n} \cap S^{0} K$ and $x B \cong K$. Thus $x \in J$ and $\eta$ is a monomorphism.

$C$ is semihereditary and $T$ is the two-sided maximal quotient ring of $C$; hence by [4, Theorem 2.3], $Z\left(T \otimes{ }_{C} T\right)=0$. Therefore by the proof of [5, Theorem 2.3], the mapping $T \otimes_{C} T \rightarrow T$ is an isomorphism. In addition, $T$ is semisimple and $T_{C}$ is flat. All of this along with the fact that ${ }_{C} B$ is flat by Lemma 3.7 yield the following sequence of monomorphisms.

$$
\begin{aligned}
\left(C^{n} / J\right) \otimes{ }_{C} B & \longrightarrow\left(T^{n} / L\right) \otimes_{C} B \longrightarrow\left(\left(T^{n} / L\right) \otimes_{T} T\right) \otimes_{C} B \\
& \longrightarrow\left(T^{n} / L\right) \otimes_{T}\left(T \otimes{ }_{C} B\right) \\
& \longrightarrow\left(T^{n} / L\right) \otimes_{T}\left(T \otimes{ }_{C} S^{0} B\right) \\
& \longrightarrow\left(T^{n} / L\right) \otimes_{T}\left(T \otimes_{C}\left(T \otimes{ }_{T} S^{0} B\right)\right) \\
& \longrightarrow\left(T^{n} / L\right) \otimes_{T}\left(\left(T \otimes_{C} T\right) \otimes_{T} S^{0} B\right) \\
& \longrightarrow\left(T^{n} / L\right) \otimes_{T}\left(T \otimes_{T} S^{0} B\right) \longrightarrow\left(T^{n} / L\right) \otimes_{T} S^{0} B .
\end{aligned}
$$

Now $\left(C^{n} / J\right) \otimes{ }_{C} B$ is isomorphic to $B^{n} / J B$ while $\left(T^{n} / L\right) \otimes{ }_{T} S^{0} B$ is isomorphic to $S^{0} B^{n} / L\left(S^{0} B\right)$. By the monomorphism of $\left(C^{n} / J\right) \otimes{ }_{0} B$ into $\left(T^{n} / L\right) \otimes{ }_{r} S^{0} B$ established in the preceeding paragraph, we have that there is a natural monomorphism $\alpha$ of $B^{n} / J B$ into $S^{0} B^{n} / L\left(S^{0} B\right)$. Now the image of $\alpha$ is $\left(B^{n}+L\left(S^{0} B\right)\right) / L\left(S^{0} B\right) \cong B^{n} /\left(B^{n} \cap L\left(S^{0} B\right)\right.$. $J B \subseteq B^{n} \cap L\left(S^{0} B\right)$; so it follows that $J B=B^{n} \cap L\left(S^{0} B\right)$.

Finally we show that $J B=K$. Since $S^{0} K$ is a direct summand of $S^{0} B^{n}$, there is an idempotent $n \times n$ matrix $p$ over $T$ such that $p\left(S^{0} B^{n}\right)=S^{0} K$. For $x \in K, x=u_{1} b_{1}+\cdots+u_{n} b_{n}$ where $u_{i} \in T^{n}, b_{i} \in$ 
$S^{0} B$. Since $p u_{i}\left(S^{0} B\right) \subseteq p\left(S^{0} B^{n}\right)=S^{0} K, p u_{i} \in L$. Now $x=p x$; so $x \in$ $L\left(S^{0} B\right) \cap B^{n}=J B$. Thus $K \subseteq J B$; so $K=J B$.

Lemma 3.9. If $N$ is a finitely generated nonsingular right $R$ module, then $N / N R_{12}$ is a projective right $R / R_{12}$-module.

Proof. Using the techniques of [17, Lemma R], N/NR $R_{12}$ can be embedded in a finite direct product of copies of $\left(S^{0} R\right) /\left(S^{0} R\right) R_{12}$. Now

$$
\left(S^{0} R\right) /\left(S^{0} R\right) R_{12}=\left[\begin{array}{cc}
S^{0} A & X \\
S^{0} B & T
\end{array}\right] /\left[\begin{array}{ll}
S^{0} A & 0 \\
S^{0} B & 0
\end{array}\right] ;
$$

so it suffices to show that $X_{C}$ and $T_{C}$ are right $R$-flat. We have already observed that $T_{C}$ is flat using condition (iii). $X_{C}$ is isomorphic to direct sum of direct summands of $T_{C}$; so $X_{C}$ is flat as $C$ is semihereditary. Hence $N / N R_{12}$ is $C$-flat as $C$ is semihereditary and hence $N / N R_{12}$ is $R / R_{12}$-projective.

The following lemma is essentially [17, Lemma T]. We include it here for completeness.

LEMmA 3.10. If $N$ is a finitely generated nonsingular right $R$-module, then $\operatorname{Tor}_{1}^{R}\left(N, R / R_{23}\right)=0$.

Proof. We will show that the mapping $N \otimes{ }_{R} R_{23} \rightarrow N$ is a monomorphism. Since $R_{23}$ is left $R$-flat by Lemma 3.7, the mapping $f: N R_{23} \otimes{ }_{R} R_{23} \rightarrow N \otimes{ }_{R} R_{23}$ is monic. Since $R_{23}$ is idempotent, $f$ is an isomorphism and it suffices to show that $N R_{23} \otimes R_{23} \rightarrow N R_{23}$ is monic.

Since $N$ is finitely generated right nonsingular, $N R_{23} \cong R_{23}^{n} / H$ for some $H \in L^{*}\left(R_{23}^{n}\right)$ and for some positive integer $n$. Then it follows that $H=\left[\begin{array}{ll}0 & 0 \\ K & J\end{array}\right]$ for some $K \in L^{*}\left(B^{n}\right)$ and $J \subseteq C^{n}$. Since $H$ is a submodule of $R_{23}^{n}, J B \subseteq K$. Given $x \in C^{n}$ such that $x B \subseteq K$, we see that $\left[\begin{array}{ll}0 & 0 \\ 0 & x\end{array}\right] R_{12} \subseteq H$. Since $R_{12}$ is essential in $R_{R},\left[\begin{array}{ll}0 & 0 \\ 0 & x\end{array}\right] \in H$ and $x \in J$. Therefore $J=\left\{x \in C^{n}: x B \subseteq K\right\}$ and by Lemma 3.8, $J B=$ $K$.

Now $H R_{23}=\left[\begin{array}{ll}0 & 0 \\ J B & J\end{array}\right]=H$; hence the mapping $H / H R_{23} \rightarrow R_{23}^{n} /$ $\left(R_{23}^{n}\right) R_{23}$ is monic. Since $R_{23}^{R}$ is right projective, $\operatorname{Tor}_{1}^{R}\left(R_{23}^{n} / H, R / R_{23}\right)=$ 0 ; i.e., $\operatorname{Tor}_{1}^{R}\left(N R_{23}, R / R_{23}\right)=0$. Thus $N R_{23} \otimes R_{23} \rightarrow N R_{23}$ is monic as required.

We now can return to the proof of the converse of Theorem 3.6.

Proof. (Converse of Theorem 3.6.) By the triangular structure 
of $R, R$ is a right nonsingular right finite dimensional ring with zero socle.

In order to show that $R$ has FGSP, we must show that $\operatorname{Ext}_{R}^{1}(N$, $W)=0$ whenever $N$ is a finitely generated right nonsingular $R$ module and $W$ is a right singular $R$-module by [6, Proposition1. 11]. Since it suffices to show that $\operatorname{Ext}_{R}^{1}\left(N, W / W R_{12}\right)=0$ and $\operatorname{Ext}_{R}^{1}(N$, $\left.W R_{12}\right)=0$, we may assume that either $W R_{12}=0$ or $W R_{23}=0$.

Case I. Let $W R_{12}=0$. Consider any short exact sequence $E: 0 \longrightarrow W \longrightarrow V \longrightarrow N \longrightarrow 0$

of right $R$-modules. Since $R / R_{12}$ is left $R$-projective, we obtain a second short exact sequence

$$
E^{*}: 0 \longrightarrow W \longrightarrow V / V R_{12} \longrightarrow N / N R_{12} \longrightarrow 0 \text {. }
$$

By Lemma 3.9, $N / N R_{12}$ is a projective right $R / R_{12}$-module; hence $E^{*}$ splits and it follows that $E$ splits.

Case II. Let $W R_{23}=0$. Consider any short exact sequence

$$
E: 0 \longrightarrow W \longrightarrow V \longrightarrow N \longrightarrow 0
$$

of right $R$-modules. By Lemma 3.10, $\operatorname{Tor}_{1}^{R}\left(N, R / R_{23}\right)=0$; so we have a second exact sequence

$$
E^{*}: 0 \longrightarrow W \longrightarrow V / V R_{23} \longrightarrow N / N R_{23} \longrightarrow 0 \text {. }
$$

Since $R_{23}$ is a two-sided ideal of $L^{*}(R)$, any singular submodule of any $R / R_{23}$-module is the same whether considered as an $R / R_{23}$-module or as an $R$-module. Hence $W$ is a singular $R / R_{23}$-module. $R / R_{23}=$ $A$ has FGSP by hypothesis; so $N / N R_{23}=Z\left(N / N R_{23}\right) \oplus U$ where $U$ is a finitely generated nonsingular $A$-module. Hence by (iv) and the fact that $A$ has FGSP, we have

$$
\begin{aligned}
& \operatorname{Ext}_{R}^{1}\left(N / N R_{23}, W\right)=\operatorname{Ext}_{A}^{1}\left(N / N R_{23}, W\right) \\
& \quad \cong \operatorname{Ext}_{A}^{1}\left(Z\left(N / N R_{23}\right), W\right) \oplus \operatorname{Ext}_{A}^{1}(U, W)=0 .
\end{aligned}
$$

Therefore $E^{*}$ splits and it follows that $E$ splits.

Certain conditions on $R$ guarantee the fact that condition (iv) of Theorem 3.6 is always satisfied. One of these conditions is that $B_{A}$ is injective. For in this case, by the proof of [17, Lemma Q], $N R_{23}$ is a direct summand of $N$ for any finitely generated right nonsingular $R$-module $N$. Hence $N / N R_{23}$ is $A$-nonsingular; so condition (iv) always holds if $A$ has FGSP. The following proposition gives a necessary and sufficient condition for $B_{A}$ to be injective. 
We will assume in this proposition that $R=\left[\begin{array}{ll}A & 0 \\ B & C\end{array}\right]$ and $A, B, C$ satisfy the conditions (i)-(iii) of Theorem 3.6.

Proposition 3.11. $B_{A}$ is injective if and only if $B$ is a left $Q^{\prime}$-module.

Proof. If $B_{A}$ is injective, then since $T=\operatorname{End}_{A}\left(S^{0} B\right)=\operatorname{End}_{A}(B)$, $B$ is a left $T$-module. Hence $B$ is a left $Q^{\prime}$-module.

Let $B$ be a left $Q^{\prime}$-module. Then by [16, Theorem 2.12] all nonsingular right $Q^{\prime}$-modules are projective. Thus the proofs of Lemmas L, M, $N$ and $O$ of [17] can be modified to fit our situation since $A_{A}$ is finite dimensional, and it follows that $B_{A}$ is injective.

The following theorem shows that a semihereditary condition on $R$ guarantees that $B_{A}$ is injective.

THEOREM 3.12. Let $R$ be a right finite dimensional semihereditary ring with zero socle and FGSP. Then $R$ is isomorphic to

$$
\left[\begin{array}{ll}
A & 0 \\
B & C
\end{array}\right]
$$

where

(i) $A$ is a right finite dimensional semihereditary semiprime ring with FGSP;

(ii) $C$ is a semihereditary ring with a two-sided Artinian two-sided classical quotient ring $Q$ such that $Q^{\prime} \subseteq T=\operatorname{End}_{A}\left(S^{0} B\right)$ and $T$ is the two-sided maximal quotient ring of $C$;

(iii) $B$ is a $Q^{\prime}-A$ bimodule such that $B_{A}$ is nonsingular.

Conversely, any such ring is a right finite dimensional semihereditary ring with zero scole such that $R$ has FGSP.

Proof. If $R$ has FGSP, then conditions (i) and (ii) follow from Theorem 3.6 and the fact that $R$ is semihereditary. By [10, Lemma 3.4], $B$ is a left $Q^{\prime}$-module since $R$ has a right Artinian classical right quotient ring by Gordon and Small [21].

Conversely, by the remarks made prior to Proposition 3.11 and by Proposition 3.11, any ring satisfying the conditions of this theorem has FGSP, is right finite dimensional and has zero socle. Now by [10, Theorem 3.7] $R$ is semihereditary since ${ }_{C} B$ is flat by Lemma 3.7 and conditions (i) and (ii).

Another ring condition which forces $B_{A}$ to be injective is the 
commutativity of $A$.

CoROLlaRy 3.13. Let $R=\left[\begin{array}{ll}A & 0 \\ B & C\end{array}\right]$ be a formal matrix ring satisfying (i)-(iii) of Theorem 3.6. If $A$ is commutative and if $R$ has FGSP, then $R$ is semihereditary.

Proof. By Theorem 3.12, we need to show that $B$ is a left $Q^{\prime}$-module. It suffices to show that $c B=B$ for all regular elements $c$ of $C$. If $c B \neq B$, then there exists $\beta \in B$ such that $c^{-1} \beta \notin B$. Since $S^{0} A$ is the classical quotient ring of $A$, there exists $\beta^{\prime} \in B$ and $b$ a regular element in $A$ such that $c^{-1} \beta=\beta^{\prime} b^{-1}$; hence $\beta b-c \beta^{\prime}=0$. Thus $\left[\begin{array}{cc}0 & 0 \\ \beta & -c\end{array}\right]\left[\begin{array}{ll}b & 0 \\ \beta^{\prime} & 0\end{array}\right]=0$.

Let $H$ be the right annihilator of $x=\left[\begin{array}{cc}0 & 0 \\ \beta & -c\end{array}\right]$ in $R . \quad H$ is a closed right ideal of $R$. For if $r \in R$ is in the closure of $H$ in $R$, then $r L \leqq H$ for some essential right ideal $L$ of $R$. Thus $x r L=0$; so $x r \in Z\left(R_{R}\right)=0$. Hence $r \in H$ and $H$ is closed.

Let $K=\left[\begin{array}{ll}b^{2} & 0 \\ \beta^{\prime} b & 0\end{array}\right] R$. Since $\left[\begin{array}{ll}b & 0 \\ \beta^{\prime} & 0\end{array}\right] \in H$, we have $K \cong H$. Consider $R / K . \quad H / K \neq 0$ as $\left[\begin{array}{ll}b & 0 \\ \beta^{\prime} & 0\end{array}\right] \in H-K$. To see this, suppose $\left[\begin{array}{ll}b & 0 \\ \beta^{\prime} & 0\end{array}\right]=$ $\left[\begin{array}{ll}b^{2} & 0 \\ \beta^{\prime} b & 0\end{array}\right]\left[\begin{array}{ll}x & 0 \\ y & z\end{array}\right] \in K$. Then $b=b^{2} x$; so $1=b x$ as $b$ is a regular element of $A$. Hence $b$ is a unit in $A$; so $c^{-1} \beta=\beta b^{-1} \in B$, a contradiction.

Now

$$
\left(\left[\begin{array}{ll}
b & 0 \\
\beta^{\prime} & 0
\end{array}\right]+K\right) / K \in Z(R / K) \subseteq H / K
$$

The second inclusion follows since $H$ is closed in $R$. The first containment follows since $b A$ is essential in $A$; so $\left[\begin{array}{ll}b & 0 \\ \beta^{\prime} & 0\end{array}\right]\left[\begin{array}{ll}b A & 0 \\ B & 0\end{array}\right] \cong K$ and $\left[\begin{array}{ll}b A & 0 \\ B & 0\end{array}\right]$ is an essential right ideal of $R$.

We now show that $Z(R / K)$ is not a direct summand of $R / K$. For if $Z(R / K)$ is a direct summand of $R / K$, then there exists $\left[\begin{array}{ll}u & 0 \\ v & 0\end{array}\right] \in H$ and $\left[\begin{array}{ll}x & 0 \\ y & z\end{array}\right] \in R$ such that

$$
\left[\begin{array}{ll}
1 & 0 \\
0 & 1
\end{array}\right]+\left[\begin{array}{ll}
b^{\prime} a & 0 \\
\beta^{\prime} b a & 0
\end{array}\right]=\left[\begin{array}{ll}
u & 0 \\
v & 0
\end{array}\right]+\left[\begin{array}{ll}
x & 0 \\
y & z
\end{array}\right] .
$$

It is immediate that $z=1$. Also $x$ is not in $H^{\prime}$ where $H^{\prime}$ is the projection of $H$ in $A$. For if $x \in H^{\prime}$, then $1 \in H^{\prime}$; so there is an $\alpha \in B$ such that $\left[\begin{array}{ll}1 & 0 \\ \alpha & 0\end{array}\right] \in H$. Then 


$$
\left[\begin{array}{cc}
0 & 0 \\
\beta & -c
\end{array}\right]\left[\begin{array}{ll}
1 & 0 \\
\alpha & 0
\end{array}\right]=\left[\begin{array}{ll}
0 & 0 \\
\beta-c \alpha & 0
\end{array}\right]=0 ;
$$

so $c^{-1} \beta=\alpha \in B$, a contradiction.

Now we show that $\left(b A+b^{2} A\right) / b^{2} A$ is essential in $A / b^{2} A$. Since $A$ is a finite direct product of Prüfer domains, the ideal lattice of $A$ is distributive by [22] or [1]. Hence if $a \in A$ is such that $\left(b^{2} A+\right.$ $a A) \cap b A=b^{2} A$, then $\left(b^{2} A \cap b A\right)+(a A+b A)=b^{2} A$ by distributivity. But $b^{2} A \cap b A=b^{2} A$; so $b^{2} A+(a A \cap b A)=b^{2} A$ and $a A \cap b A \cong b^{2} A$. Thus $b a=b^{2} a^{\prime}$ for $a^{\prime} \in A$; so $a=b a^{\prime}$. Hence $a A \leqq b A$. Consequently $\left(b A+b^{2} A\right) / b^{2} A$ is essential in $A / b^{2} A$.

Since $x+b^{2} A \notin\left(b A+b^{2} A\right) / b^{2} A$, there is a regular element $d$ of $A$ such that $x d+b^{2} A$ is a nonzero element of $\left(b A+b^{2} A\right) / b^{2} A$. As $z=1$, there exists $\left[\begin{array}{ll}d & 0 \\ w & 0\end{array}\right]$ such that $\left[\begin{array}{ll}x & 0 \\ y & z\end{array}\right]\left[\begin{array}{ll}d & 0 \\ w & 0\end{array}\right] \in\left[\begin{array}{ll}b & 0 \\ \beta^{\prime} & 0\end{array}\right] R / K$. Thus $\left(\left[\begin{array}{ll}x & 0 \\ y & z\end{array}\right]+K\right) R$ has nonzero intersection with $Z(R / K)$ which contradicts the assumption that $Z(R / K)$ is a direct summand of $R / K$. Thus we conclude that if $R$ has FGSP, $B$ is a left $Q^{\prime}$-module.

If $R$ satisfies Theorem 3.6, $B_{A}$ need not be injective, for let $A$ be a two-sided Noetherian prime ring with zero socle such that every singular right $A$-module is injective; that is, $A$ is a $S I$-ring in the sense of Goodearl [16]. Then $A$ has a two-sided classical (maximal) quotient ring and $A$ is hereditary by Goodearl [16, Proposition 3.3]. Examples of such rings are given by Cozzens [7]. Then the ring $\left[\begin{array}{cc}A & 0 \\ A & A\end{array}\right]$ satisfies Theorem 3.6 but $A_{A}$ is not injective.

We now investigate the situation when $R=\left[\begin{array}{ll}A & 0 \\ B & C\end{array}\right]$ satisfies (i)(iii) of Theorem 3.6 and $A$ is a two-sided hereditary Noetherian prime (HNP) ring. Let $\sigma$ be an idempotent kernel functor for the category of right $A$-modules such that $A$ is $\sigma$-torsion free; i.e., $\sigma(A)=0$. Then $\sigma$ has property $T$ by Goldman [12, Theorem 4.4 and Theorem 4.5]. This means that if $Q_{\sigma}$ is the ring of quotients of $A$ relative to $\sigma$ and $Q_{\sigma}(M)$ is the module of quotients for an $A$ module $M$, then $Q_{\sigma}(M) \cong M \otimes Q_{\sigma}$. A right $A$-module $E$ is $\sigma$-injective if it has the property: if $M$ is any right $A$-module and $N$ is a submodule of $M$ such that $M / N$ is $\sigma$-torsion, then every $A$-homomorphism from $N$ to $E$ extends to a homomorphism of $M$ to $E$. Any $Q_{\sigma}$-module is $\sigma$-injective by [12, Theorem 4.3]. For further details see [12] or [30].

Let $\mathscr{C}=\left\{S: S\right.$ is a simple right $A$-module and $\operatorname{Ext}_{A}^{1}=\left(S, S_{1}\right) \neq$ 0 for some simple right $A$-module $S_{1}$ \} and let $\sigma$ be the torsion theory generated by the family $\mathscr{C}$. With this notation, we have the following proposition. 
Proposition 3.14. Let $R=\left[\begin{array}{cc}A & 0 \\ B & C\end{array}\right]$ satisfy conditions (i)-(iii) of Theorem 3.6 and let $A$ be a HNP ring. If $R$ has FGSP, then $B=Q_{a}(B)$.

Proof. We need to show that $S^{0} B / B$ is $\sigma$-torsion free; that is, $S^{0} B / B$ has no simple submodule isomorphic to $S \in \mathscr{C}$. For if $S^{0} B / B$ contains such a simple submodule, then there exists a $\beta \in S^{0} B / B$ such that $\beta J \subseteq B$ for $J$ a maximal right ideal of $A$ such that $A / J \cong S \in$ C. $J \subseteq\{a \in A: \beta a \in B\}$ and $\{a \in A: \beta a \in B\}$ is a proper right ideal of $A$; so $J=\{a \in A: \beta a \in B\}$.

Consider

$$
H=\left\{\left[\begin{array}{ll}
a & 0 \\
\beta a & 0
\end{array}\right]: a \in J\right\}
$$

$H$ is a closed right ideal of $R$. For suppose that $\left[\begin{array}{cc}u & 0 \\ v & w\end{array}\right]\left[\begin{array}{ll}K & 0 \\ L & 0\end{array}\right] \subseteq H$ where $K_{A}$ is essential in $A_{A}$ and $L_{A}$ is essential in $B_{A}$. Then $w L=0$. Since $L_{A}$ is essential in $B_{A}$ and $w \in \operatorname{End}_{A}\left(S^{0} B\right), w\left(S^{0} B\right)=0$. Hence $w=0$. Thus $\left[\begin{array}{ll}u & 0 \\ v & 0\end{array}\right]\left[\begin{array}{ll}K & 0 \\ L & 0\end{array}\right] \cong H$; so

$$
\left[\begin{array}{ll}
u & 0 \\
v & 0
\end{array}\right]\left[\begin{array}{ll}
k & 0 \\
0 & 0
\end{array}\right]=\left[\begin{array}{ll}
u k & 0 \\
v k & 0
\end{array}\right] \in H
$$

for $k$ a regular element of $K$. Thus $\beta u k=v k$; so $\beta u=v$ as $k$ is a regular element of $K$. Thus $\left[\begin{array}{ll}u & 0 \\ v & 0\end{array}\right]=\left[\begin{array}{ll}u & 0 \\ \beta u & 0\end{array}\right] \in H$.

Let $N=R / H$. Then $N / N R_{23} \cong A / J \cong S$. Since $R$ has FGSP, $0=\operatorname{Ext}_{A}^{1}\left(N / N R_{23}, S_{1}\right) \cong \operatorname{Ext}_{A}^{1}\left(A / J, S_{1}\right)$ for all simple right $R$-modules $S_{1}$ by Theorem 3.6 (iv). This is a contradiction; so $B=Q_{o}(B)$.

We have the following partial converse of Proposition 3.14.

Lemma 3.15. Let $R=\left[\begin{array}{ll}A & 0 \\ B & C\end{array}\right]$ satisfy conditions (i)-(iii) of Theorem 3.6 and $A$ be a $H N P$ ring. If $B=Q_{\sigma}(B)$, then $N / N R_{23}$ is $\sigma$-torsion free for any finitely generated nonsingular right $R$ module $N$.

Proof. If $N / N R_{23}$ is not $\sigma$-torsion free, then there is a submodule $N^{\prime}$ of $N$ such that $N R_{23} \cong N^{\prime} \cong N$ and $N^{\prime} / N R_{23} \cong S \in \mathscr{C}$. Since $R_{23}$ is a direct summand of $R_{R}$ and $N$ is finitely generated, then $N R_{23}$ is finitely generated. Therefore $N^{\prime}$ can be chosen to be finitely generated. 
Let $N^{\prime}=F / H$ where $F=R^{n}$ for some positive integer $n$ and $H \in L^{*}(F) . \quad H=\left[\begin{array}{ll}U & 0 \\ V & J\end{array}\right]$ where $U \subseteq A^{n}, V \cong B^{n}$ and $J \subseteq C^{n} . \quad F / H$ is nonsingular; so $\mathrm{Fe} / \mathrm{He}$ is nonsingular where $e=\left[\begin{array}{ll}1 & 0 \\ 0 & 0\end{array}\right]$. For if $\left[\begin{array}{ll}u & 0 \\ v & 0\end{array}\right] L \subseteq H e \subseteq H$ where $L$ essential in $R_{R}$, then $\left[\begin{array}{ll}u & 0 \\ v & 0\end{array}\right] \in H$; so $\left[\begin{array}{ll}u & 0 \\ v & 0\end{array}\right] \in H e$. Thus we may assume that $H=\left[\begin{array}{ll}U & 0 \\ V & 0\end{array}\right]$.

Let $Z=\left\{\left[\begin{array}{ll}0 & 0 \\ v & 0\end{array}\right]:\left[\begin{array}{ll}0 & 0 \\ v & 0\end{array}\right] \in H\right\} . \quad Z$ is a closed submodule of $F$ since $Z=H \cap F R_{23}$ and both $H$ and $F R_{23}$ are closed in $F$. Since $A^{n} / U \cong S$, pick $y \in A^{n} / U$ and set $J=\{a \in A: u a \in U\}$. Then $A / J \cong$ $A^{n} / U \cong S$. For $a \in J$, define $f(a)=v+Z$ where $\left[\begin{array}{ll}y a & 0 \\ v & 0\end{array}\right] \in H$. By the definition of $Z, f$ is a well defined homomorphism of $J$ into $V / Z \subseteq$ $B^{n} / Z . \quad B^{n}$ is $\sigma$-injective and $B^{n} / Z$ is $\sigma$-torsion free since $F / Z$ is nonsingular. Hence by [12, Theorem 4.5$], B^{n} / Z$ is $\sigma$-injective; so $f$ can be extended to $A$. Therefore $f$ is given as a left multiplication by an element $x \in B^{n} / Z$. Thus for $a \in J, f(a)=(x+Z) a \in V / Z$. Therefore $\left(\left[\begin{array}{ll}y & 0 \\ x & 0\end{array}\right]+Z\right) J \subseteq H / Z$. $y \in U$; so $\left[\begin{array}{ll}y & 0 \\ x & 0\end{array}\right]+Z \in H / Z$. But

$$
\left[\begin{array}{ll}
y & 0 \\
x & 0
\end{array}\right]+Z \in Z((F / Z) /(H / Z))
$$

since

$$
\left(\left[\begin{array}{ll}
y & 0 \\
x & 0
\end{array}\right]+Z\right)\left[\begin{array}{ll}
J & 0 \\
B & 0
\end{array}\right] \subseteq H / Z
$$

But $(F / Z) /(H / Z) \cong F / H$ is nonsingular, a contradiction. Thus $N / N R_{23}$ is $\sigma$-torsion free.

Given a right ideal $M$ in a ring $T$, the idealizer of $M$ in $T$ is the largest subring of $T$ which contains $M$ as a two-sided ideal. Let $A$ be the idealizer of a HNP $S I$-ring $A^{*}$ relative to a semimaximal right ideal of $A^{*}$ (see [14] or [25] for details). By Goodearl [14, Theorem 9] $A$ is a splitting ring. It follows from [25, Corollary 2.4] that if $U$ is a simple right $A$-module, then there is a maximal right ideal $M$ of $A^{*}$ such that $U$ is one of the following three types: (I) $U \cong A^{*} / M$; (II) $U \cong A^{*} /(A+M)$ or (III) $U \cong(A+$ $M) / M$. Let $X=\{S: S$ is a simple right $A$-module of type I or III $\}$. Then $A^{*}=Q_{\tau}$ where $\tau$ is the idempotent kernel functor generated by the cyclic modules of the form $A / I$ where $A / I$ has a composition series with no composition factors isomorphic to $S \in X$. For details see [14].

It can be checked that the only nonsplit exact sequence of right 
$A$-modules

$$
0 \longrightarrow S_{1} \longrightarrow Y \longrightarrow S \longrightarrow 0
$$

where $S_{1}$ and $S$ are simple $A$-modules occurs where $S_{1}$ has type III and $S$ has type II. Hence a simple $A$-module $S \in \mathscr{C}$ if and only if $S$ is $\tau$-torsion. We now have the following proposition.

Proposition 3.16. Let $R=\left[\begin{array}{ll}A & 0 \\ B & C\end{array}\right]$ which satisfies conditions (i)(iii) of Theorem 3.6. If $A$ is an idealizer of a HNP SI-ring $A^{*}$ relative to a semimaximal right ideal of $A^{*}$, then $R$ has FGSP if and only if $B=Q_{\sigma}(B)$.

Proof. If $R$ has FGSP, then $Q_{o}(B)=B$ by Proposition 3.14.

Conversely, let $Q_{\sigma}(B)=B$. We need to show that the exact sequence of right $R$-modules

$$
E: 0 \longrightarrow W \longrightarrow V \longrightarrow N \longrightarrow 0
$$

is split exact where $V$ is finitely generated and $W=Z\left(V_{R}\right)$. As in the proof of the converse of Theorem 3.6, we may assume $W R_{12}=0$ or $W R_{23}=0$. The proof of the case when $W R_{12}=0$ is identical to that given in the proof of Theorem 3.6. So assume that $W R_{23}=0$. Lemma 3.10 is still valid; hence we have a second short exact sequence

$$
E^{*}: 0 \longrightarrow W \longrightarrow V / V R_{23} \longrightarrow N / N R_{23} \longrightarrow 0 \text {. }
$$

Since $A \cong R / R_{23}$ is a HNP ring, $W$ has a composition series. Hence we can further assume that $W$ is a simple singular $A$-module. By Lemma 3.15, $N / N R_{23}$ is $\sigma$-torsion free; so by the remarks made prior to this proposition, $N / N R_{23}$ is $\tau$-torsion free. Thus $0 \rightarrow$ $N / N R_{23} \rightarrow Q_{\tau}\left(N / N R_{23}\right)$ is monic; hence $\operatorname{Ext}_{A}^{1}\left(Q_{\tau}\left(N / N R_{23}\right), W\right) \rightarrow \operatorname{Ext}_{A}^{1}(N /$ $\left.N R_{23}, W\right) \rightarrow 0$ is exact. Hence if $\operatorname{Ext}_{A}^{1}\left(Q_{\tau}\left(N / N R_{23}\right), W\right)=0$, then $\operatorname{Ext}_{A}^{1}\left(N / N R_{23}, W\right)=0$.

Since $W$ is $A$-singular and $Q_{=}=A^{*}$ is nonsingular, $\operatorname{Ext}_{A}^{1}\left(A^{*}, W\right)=$ 0 as $A$ is a splitting ring. Hence by [2, Proposition 4.14, p. 118], $\operatorname{Ext}_{A}^{1}\left(Q_{\tau}\left(N / N R_{23}\right), W\right) \cong \operatorname{Ext}_{A}^{1} *\left(Q_{\tau}\left(N / N R_{23}\right), \operatorname{Hom}_{A}\left(A^{*}, W\right)\right)$. Since all the faithful simple $A$-modules are injective by [14, Theorem 9], we can assume that $W$ is bounded; say $W I=0$ for a nonzero ideal $I$ of $A$. As $A$ is the idealizer of $A^{*}$, we may assume that $I$ is a right ideal of $A^{*}$ and $I^{2}=I$ by $\left[14\right.$, Lemma D]. $\operatorname{Hom}_{A}\left(A^{*}, W\right)$ is a right $A^{*}$-module via $(f a)(x)=f(a x)$ for $a, x \in A^{*}$. Thus for $f \in$ $\operatorname{Hom}_{A}\left(A^{*}, W\right)$, we have 


$$
(f I)(x)=f(I x) \leqq f(I)=f\left(I^{2}\right)=f(I) I=0 .
$$

Therefore $\operatorname{Hom}_{A}\left(A^{*}, W\right)$ is a bounded $A^{*}$-module. But $A^{*}$ is a simple ring; hence $\operatorname{Hom}_{A}\left(A^{*}, W\right)=0$. Thus it follows that $\operatorname{Ext}_{A}^{1}\left(Q_{\tau}(N)\right.$ $\left.\left.N R_{23}\right), W\right)=0$. But by the previous paragraph, $\operatorname{Ext}_{A}^{1}\left(N / N R_{23}, W\right)=$ 0 and $E^{*}$ splits. Thus $E$ is a split exact sequence and $R$ has FGSP.

Letting $A$ and $A^{*}$ be as in Proposition 3.16, where $A \neq A^{*}$ it follows that $\left[\begin{array}{cc}A & 0 \\ A & A\end{array}\right]$ does not have FGSP whereas $\left[\begin{array}{cc}A & 0 \\ A^{*} & A\end{array}\right]$ has FGSP.

4. Essential products of rings having FGSP. Given two right nonsingular rings $R_{1}$ and $R_{2}$, an essential product of $R_{1}$ and $R_{2}$ is any subdirect product $R$ of $R_{1}$ and $R_{2}$ which contains an essential right ideal of $R_{1} \times R_{2}$. Goodearl has shown [13, Theorem 3.10] that any right nonsingular ring $R$ is an essential product of nonsingular rings $R_{1}$ and $R_{2}$ where $R_{1}$ has essential socle and $R_{2}$ has zero socle. In this section we shall characterize right nonsingular rings $R$ with FGSP which either are right finite dimensional or possess a semiprimary classical right quotient ring in terms of an essential product of $R_{1}$ which has essential socle and $R_{2}$ which has zero socle.

Throughout this section we shall assume that the right nonsingular ring $R$ is an essential product of right nonsingular rings $R_{1}$ and $R_{2}$ where $R_{1}$ has essential socle and $R_{2}$ has zero socle. Then $S^{0} R=S^{0} R_{1} \times S^{0} R_{2}$ by [13, Proposition 2]; so let $\pi_{i}: S^{0} R \rightarrow S^{0} R_{i}$ be the natural projection maps for $i=1$, 2. Then $\pi_{i}(R)=R_{i}$ for $i=1$, 2 and we set $E_{1} \times 0=R \cap\left(R_{1} \times 0\right)$ and $0 \times E_{2}=R \cap\left(0 \times R_{2}\right) . \quad E_{1}$ and $E_{2}$ are two-sided ideals of $R_{1}$ and $R_{2}$ respectively and they are essential as right ideals. We begin with the following lemma.

LeMma 4.1. $R_{1}$ and $R_{2}$ have FGSP if $R$ has FGSP.

Proof. $\quad R_{1} \cong R /\left(0 \times E_{2}\right)$ and $0 \times E_{2}$ is a closed right ideal of $R$ by [13, Propositions 7 and 8]; hence by [16, Proposition 1.11], $R_{1}$ has FGSP. Similarly $R_{2}$ has FGSP.

If $R_{R}$ is finite dimensional, then $R_{1}$ and $R_{2}$ are right finite dimensional rings. If, in addition, $R$ has FGSP, by Theorems 2.5 and 3.6 we have that

$$
R_{1} \cong\left[\begin{array}{ll}
A_{1} & 0 \\
B_{1} & C_{1}
\end{array}\right] \text { and } R_{2} \cong\left[\begin{array}{cc}
A_{2} & 0 \\
B_{2} & C_{2}
\end{array}\right]
$$

where the $A_{i}$ 's, $B_{i}$ 's, and $C_{i}$ 's satisfy the conditions of the respective 
theorems. Let $e_{i}=\left[\begin{array}{ll}1 & 0 \\ 0 & 0\end{array}\right]$ where 1 is the identity of $A_{i}$ for $i=1,2$ and $f_{i}=\left[\begin{array}{ll}0 & 0 \\ 0 & 1\end{array}\right]$ where 1 is the identity of $C_{i}$ for $i=1,2$. Then $R_{1} e_{1}=\operatorname{soc}\left(R_{1}\right)=\operatorname{soc}(R)$ as $R_{2}$ has zero socle. Hence $R_{1} e_{1} \subseteq E_{1}$; so $e_{1} \in R$. Thus we have

LemMA 4.2. $e_{1} \in R$.

The following lemma and its proof is contained in [13, Theorem 12].

Lemma 4.3. Let $R$ have FGSP. Then the following statements hold.

(a) If $L_{i}$ is any essential right $R_{i}$-submodule of $E_{i}$, then $E_{\imath} / L_{i}$ is a direct summand of $R_{i} / L_{i}$ for $i=1,2$.

(b) $E_{i}^{2}=E_{i}$ for $i=1,2$.

Let $E_{i}^{\prime}=f_{i} E_{\imath} f_{i}$ for $i=1,2$. Then we have the following lemma.

Lemma 4.4. Let $R$ have FGSP. Then $E_{1}^{\prime}$ is a direct summand of $C_{1}$ as a right ideal and $E_{2}^{\prime}=C_{2}$.

Proof. Let $e=\left(e_{1}, e_{2}\right)$ and $f=\left(f_{1}, f_{2}\right)$. Then $e$ and $f$ are idempotents of $R_{1} \times R_{2}$. Since $R_{1} e_{1}$ is essential in $R_{1}$ as a right ideal and $R_{2} e_{2}$ is essential in $R_{2}$ as a right ideal $\left(R_{1} \times R_{2}\right) e \cap R$ is an essential right ideal of $R$. Let $M=R \cap f\left(R_{1} \times R_{2}\right)$. Since ef $=0$, it follows easily that $M$ is a two-sided ideal of $L^{*}(R)$ whose left annihilator is an essential right ideal of $R$. By the proof of Lemma 3.1, $M^{2}=g R$ for $g$ an idempotent element of $R$. Let $\pi_{1}(g)=g_{1}$ and $\pi_{2}(g)=g_{2}$. Let $E=E_{1} \times E_{2}$ and $E^{\prime}=E \cap\left[f\left(R_{1} \times R_{2}\right) f\right] . \quad\left(E^{\prime}\right)^{2}=E^{\prime}$ since $E^{2}=E$ by Lemma 4.3. $E^{\prime} \subseteq M$; so $E^{\prime}=\left(E^{\prime}\right)^{2} \subseteq M^{2}=g R$. Let $E_{1}^{*}=\left(g_{1} R g_{1} \times 0\right) \cap g R g=g_{1} E_{1}^{\prime} g_{1}$ and $E_{2}^{*}=\left(0 \times g_{2} R g_{2}\right) \cap g R g=$ $g_{2} E_{2}^{\prime} g_{2}$. Since $R, R_{1}$, and $R_{2}$ are finite dimensional rings, the techniques used in the proof of Lemma 3.3 can be applied to show that $g R g, g_{1} R_{1} g_{1}$, and $g_{2} R_{2} g_{2}$ are all semihereditary rings. Now $g R$ is a two-sided ideal of $R$; so $R \cong\left[\begin{array}{ll}g^{\prime} R g^{\prime} & 0 \\ g R g^{\prime} & g R g\end{array}\right]$ when $g^{\prime}=1-g$ and, in addition, $R g^{\prime}$ is an essential right ideal of $R$. Thus by Lemma 2.2, $g\left(S^{0} R\right) g$ is a right flat overring of $g R g$. Similarly $g_{1}\left(S^{0} R\right) g_{1}$ and $g_{2}\left(S^{0} R\right) g_{2}$ are right flat overrings of $g_{1} R_{1} g_{1}$ and $g_{2} R_{2} g_{2}$ respectively. $g\left(S^{0} R\right) g=g_{1}\left(S^{0} R\right) g_{1} \times g_{2}\left(S^{0} R\right) g_{2}$; so by [9, Proposition 3.8], $E_{1}^{*}$ and $E_{2}^{*}$ are direct summands of $g_{1} R g_{1}$ and $g_{2} R g_{2}$ respectively as right ideals.

Let $g_{1}^{\prime}=f_{1} g_{1} f_{1}$. Since $C_{1}$ is a semihereditary ring with a semi- 
primary classical left quotient ring by Theorem 2.5, $C_{1}$ has a complete set of orthogonal idempotents $\left\{h_{1}, \cdots, h_{n}\right\}$ such that $g_{1}^{\prime} C_{1}=$ $\sum_{i>k} h_{i} C_{1}$ by [9, Lemma 3.3]. This implies that $\left\{h_{k+1}, \cdots, h_{n}\right\}$ is a complete set of orthogonal idempotents for $g_{1}^{\prime} C_{1} g_{1}^{\prime}$. By a similar argument $E_{1}^{*}=\sum_{i>l} h_{i}\left(g_{1}^{\prime} C_{1} g_{1}^{\prime}\right)$ where $l \geqq k$. Claim $E_{1}^{\prime}=\sum_{i>l} h_{i} C_{1}$. Let $X=E_{1}^{\prime} \cap h_{j} C_{1}$ where $j \leqq l$. Note the $j>k$ since $E_{1}^{\prime} \leqq g_{1}^{\prime} C_{1}$. Now $X \subseteq h_{j} N\left(C_{1}\right)$ since $E_{1}^{*}$ is a direct summand of $g_{1}^{\prime} C_{1} g_{1}^{\prime}$. If $X \neq 0$, then $L=h_{j} R_{1} e_{1}$ is a proper essential $R_{1}$-submodule of $h_{j} R_{1}$ and $(X+L) / L$ is a direct summand of $h_{j} R_{1} / L$ by Lemma 4.3. But this is impossible as $(X+L) / L \cong\left(h_{j} N\left(C_{1}\right)+L\right) / L$ and $(X+L) / L$ is a small submodule of $h_{j} R_{1} / L$. Hence $X=0$ and $E_{1}^{\prime}=\sum_{i>l} h_{i} C_{1}$.

Now let $g_{2}^{\prime}=f_{2} g_{2} f_{2}$. By arguments similar to those given in the previous paragraph, $C_{2}$ has a complete set of orthogonal idempotents $\left\{h_{1}^{\prime}, \cdots, h_{m}^{\prime}\right\}$ such that $E_{2}^{\prime}=\sum_{i>t} h_{i}^{\prime} C_{2}$. Claim $E_{2}^{\prime}=C_{2}$. For if $E_{2}^{\prime} \neq C_{2}$, then $h_{j}^{\prime} \notin E_{2}^{\prime}$ for some $j \leqq t$. Since $R_{2}$ has zero socle, $h_{j}^{\prime} E_{2}$ contains a proper essential right submodule $H$ by [16, Proposition 1.2]. Let $L=e_{2} E_{2} \oplus h_{1}^{\prime} E_{2} \oplus \cdots \oplus h_{j-1}^{\prime} E_{2} \oplus H \oplus h_{j+1}^{\prime} E_{2} \oplus \cdots \oplus$ $h_{m}^{\prime} E_{2}$. $L$ is a proper essential submodule of $E_{2}$. Thus $E_{2} / L$ is a direct summand of $R_{2} / L$ by Lemma 4.3. However, $E_{2} / L$ is exactly $h_{j}^{\prime} E_{2} e_{2} / H$; hence $h_{j}^{\prime} E_{2} e_{2} / H$ is a direct summand of $h_{j}^{\prime} R_{2} / H$. But this is impossible since $h_{j}^{\prime} E_{2} e_{2} \subseteq h_{j}^{\prime} N\left(R_{2}\right)$ which forces $h_{j}^{\prime} E_{2} e_{2} / H$ to be a small submodule of $h_{j}^{\prime} R_{2} / H$.

If $R=\left[\begin{array}{ll}A & 0 \\ B & C\end{array}\right]$ is a formal triangular matrix ring satisfying Theorem 2.5, we shall say that $R$ satisfies $(\alpha)$. If $R=\left[\begin{array}{ll}A & 0 \\ B & C\end{array}\right]$ is a formal triangular matrix ring satisfying Theorem 3.6 , we shall say that $R$ satisfies $(\beta)$. With this notation, we have the first main result of this section.

THEOREM 4.5. Let $R$ be a right nonsingular right finite dimensional ring with FGSP. Then $R$ is a subdirect product of $R_{1}$ and $R_{2}$ where

$\begin{aligned} \text { (1) } R_{1}= & {\left[\begin{array}{cc}A_{1} & 0 \\ B_{1} & C_{1}\end{array}\right]=\left[\begin{array}{c:cc}A_{11} & 0 & 0 \\ \hdashline A_{21} & A_{22} & 0 \\ A_{31} & A_{32} & A_{33}\end{array}\right] } \\ & \text { satisfies }(\alpha) \text { and } E_{1}=\left[\begin{array}{ccc}A_{11} & 0 & 0 \\ A_{21} & 0 & 0 \\ A_{31} & A_{32} & A_{33}\end{array}\right] ;\end{aligned}$

(2) $\quad R_{2}=\left[\begin{array}{ll}A_{2} & 0 \\ B_{2} & C_{2}\end{array}\right]$ satisfies $(\beta)$ and $E_{2}=\left[\begin{array}{ll}X & 0 \\ B_{2} & C_{2}\end{array}\right]$ where $X$ is a two-sided idempotent ideal of $A_{2}$ essential as a right ideal having the property: if $L$ is an essential submodule of the right $A_{2}$-module 
$X$, then $X / L$ is a direct summand of $A_{2} / L$.

Conversely, any subdirect product of ring $R_{1}$ and $R_{2}$ satisfying (1) and (2) is a right nonsingular right finite dimensional ring with FGSP.

Proof. If $R$ has FGSP, then $R_{1}$ and $R_{2}$ satisfy (1) and (2) by Lemmas $4.1,4.2,4.3$, and 4.4 .

To prove the converse we will need to prove some lemmas. We shall assume that $R$ is an essential product of $R_{1}$ and $R_{2}$ satisfying (1) and (2) in Lemmas 4.6 and 4.7.

Lemma 4.6. If $N$ is a finitely generated nonsingular right $R$ module, then there is an exact sequence of $R$-modules

$$
0 \longrightarrow N_{1} \longrightarrow N \longrightarrow N_{2} \longrightarrow 0
$$

where $N_{1}$ is a finitely generated nonsingular right $R_{1}$-module and $N_{2}$ is a finitely generated nonsingular right $R_{2}$-module.

Proof. Given any nonsingular $R$-module $N$, there is an exact sequence of $R$-modules

$$
0 \longrightarrow N_{1} \longrightarrow N \longrightarrow N_{2} \longrightarrow 0
$$

where $N_{i}$ is $R_{i}$-nonsingular for $i=1,2$ by [13, Proposition 3]. Thus if $N$ is finitely generated as well, $N_{2}$ is a finitely generated nonsingular $R_{2}$-module.

It remains to show that $N_{1}$ is finitely generated. First we will show that $N_{2}$ is AFR as an $R$-module. Since $N_{2}$ is a finitely generated nonsingular $R_{2}$-module and $R_{2}$ has FGSP, $N_{2}$ is AFR as an $R_{2}$-module by [11, Corollary 2]. Let $H=\operatorname{soc}\left(R_{R}\right)$ and $S=R / H$. By [16, page 65] it is enough to show that $N_{2}$ is finitely related as an $S$-module. Now $E_{1}^{\prime}=E_{1} \cap f_{1} R_{1} f_{1}$ is a direct summand of $S$ as a right ideal. Furthermore $S / E_{1}^{\prime} \cong R_{2}$. Since $N_{2}$ is AFR as a $R_{2}$ module and $R_{2}$ has zero socle, there is an exact sequence of $R_{2}$ modules

$$
0 \longrightarrow K \longrightarrow R_{2}^{n} \longrightarrow N_{2} \longrightarrow 0
$$

where $n$ is a positive integer and $K$ is finitely generated. Then there exists an exact sequence

$$
0 \longrightarrow K \oplus\left(E_{1}^{\prime}\right)^{n} \longrightarrow\left(R_{2} \oplus E_{1}^{\prime}\right)^{n} \longrightarrow N_{2} \longrightarrow 0
$$

and $N_{2}$ is finitely related as an $S$-module. Thus $N_{2}$ is AFR as an $R$-module. 
By [16, Lemma 4.7(a)] $N_{1}$ is AFG as an $R$-module. Since $N_{1}$ is a submodule of $N$ and $\operatorname{soc}\left(N_{R}\right)$ is finitely generated, $N_{1}$ is finitely generated.

The following lemma is due to Goodearl and using the fact that $N$ is finitely generated, the proof is virtually identical to the proof of Case II of [13, Theorem 12, page 501].

LEMMA 4.7. Let $N$ be a finitely generated right nonsingular $R_{i}$-module and $W$ a singular right $R_{j}$-module for $i \neq j$. Then $\operatorname{Ext}_{R}^{1}(N, W)=0$.

We now return to the proof of the converse of Theorem 4.5.

Proof. (Converse of Theorem 4.5.) That $R$ is right nonsingular and right finite dimensional follows from the fact that $R$ is an essential product of $R_{1}$ and $R_{2}$. To show that $R$ has FGSP, it is sufficient to show that any exact sequence of right $R$-modules

$$
0 \longrightarrow W \longrightarrow Y \longrightarrow N \longrightarrow 0
$$

is split exact where $W$ is $R$-singular and $N$ is finitely generated nonsingular. By [13, Proposition 3] and Lemma 4.6 there are exact sequences of $R$-modules

$$
\begin{aligned}
& 0 \longrightarrow W_{1} \longrightarrow W \longrightarrow W_{2} \longrightarrow 0 \\
& 0 \longrightarrow N_{1} \longrightarrow N \longrightarrow N_{2} \longrightarrow 0
\end{aligned}
$$

where $W_{i}$ is $R_{i}$-singular for $i=1,2$ and $N_{i}$ is finitely generated $R_{i}$-nonsingular for $i=1,2$.

To show that $\operatorname{Ext}_{R}^{1}(N, W)=0$ it suffices to show that $\operatorname{Ext}_{R}^{1}\left(N_{i}\right.$, $\left.W_{j}\right)=0$ for $i=1,2$ and $j=1,2$. Now $\operatorname{Ext}_{R}^{1}\left(N_{i}, W_{j}\right)=0$ for $i \neq j$ by Lemma 4.7 .

Consider an exact sequence

$$
D: 0 \longrightarrow W_{1} \longrightarrow Y \longrightarrow N_{1} \longrightarrow 0
$$

of $R$-modules. Since $W_{1}$ and $N_{1}$ are $R_{1}$-modules and $E_{2}^{2}=E_{2}, D$ is an exact sequence of $R_{1}$-modules. Thus $D$ splits as $R_{1}$-modules since $R_{1}$ has FGSP. From this it follows that the sequence splits as $R$ modules so $\operatorname{Ext}_{R}^{1}\left(N_{1}, W_{1}\right)=0$.

By a similar argument, $\operatorname{Ext}_{R}^{1}\left(N_{2}, W_{2}\right)=0$. Thus $R$ has FGSP.

Goodearl has given an example in [13, Example 1, page 503] which shows that the essential product given in Theorem 4.5 need 
not be a direct product. In fact Theorem 4.5 shows that the example given there is the "canonical" way to construct essential products which are not direct products. In order to construct an essential product as described in Theorem 4.5 it is necessary that the ring $A_{2}$ have a two-sided idempotent ideal $X$ essential as a right ideal such that if $L$ is an essential submodule of the right $A_{2}$ module $X, X / L$ must be a direct summand of $A_{2} / L$. Proposition 4.8 gives a class of rings possessing such an ideal.

A subring $R$ of $T$ is an iterated idealizer from $T$ provided there is a chain of subrings $R_{0}=R \cong R_{1} \subseteq \cdots \subseteq R_{n}=T$ such that each $R_{i}$ is the idealizer of a semimaximal right ideal of $R_{i+1}$.

Proposition 4.8. Let $A$ be a HNP ring which is an iterated idealizer of a HNP SI-ring. Then A has a minimal two-sided ideal $M$ which is idempotent and has the property that if $L$ is an essential submodule of $M_{A}, M / L$ is a direct summand of $A / L$.

Proof. By [14, Lemma D] $A$ possesses a minimal two-sided ideal $M$ which is idempotent. First we show that $\operatorname{Ext}_{A}^{1}(A / M, S)=0$ for all simple right $A$-modules $S$. By [14, Theorem 10], if $S$ is a faithful simple $A$-module, then $A$ injective; so $\operatorname{Ext}_{A}^{1}(A / M, S)=0$. If $S$ is bounded, that is, if $r_{A}(S) \neq 0$, and if $\operatorname{Ext}_{A}^{1}(A / M, S) \neq 0$, then there is an exact sequence

$$
0 \longrightarrow S \longrightarrow X \longrightarrow A / M \longrightarrow 0
$$

which is nonsplit and $X$ can be taken to be cyclic. Since $A / M$ and $S$ are both bounded, $X$ is bounded as $A$ is a prime ring. Thus $M X=0$ as $M$ is the minimal two-sided ideal of $A$. Therefore $X$ is a homomorphic image of $A / M$; so $X$ has composition series length less than or equal to that of $A / M$. But this is impossible; so $\operatorname{Ext}_{A}^{1}(A / M, S)=0$. It follows that $\operatorname{Ext}_{A}^{1}(A / M, W)=0$ for all finitely generated singular $A$-modules $W$.

Hence if $L$ is an essential submodule of $M_{A}$, then $\operatorname{Ext}_{A}^{1}(A / M$, $M / L)=0$; so that $M / L$ is a direct summand of $A / L$.

We now turn to studying rings $R$ with FGSP which possess a semiprimary classical right quotient ring $Q$. Let $R_{1}, R_{2}, \pi_{1}, \pi_{2}, E_{1}$, and $E_{2}$ be as defined in the beginning of this section. Since $R$ is right nonsingular, $Q$ is right nonsingular and $\operatorname{soc}\left(Q_{Q}\right)=Q e$ where $e$ is an idempotent element of $Q$. Set $\pi_{i}(Q)=Q_{i}, \pi_{i}(e)=e_{i}$ and $\pi_{i}(1-e)=f_{i}$ for $i=1,2$. Then

$$
Q_{i}=\left[\begin{array}{cc}
e_{i} Q_{i} e_{i} & 0 \\
f_{i} Q_{i} e_{i} & f_{i} Q_{i} f_{i}
\end{array}\right]
$$


for $i=1,2$. By Lemma $4.2, e_{1} \in R$ as $R_{1}$ has essential socle and $R_{2}$ has zero socle. Thus it follows that $f_{1} \in R_{1}$. Furthermore, we have the following lemma in terms of this notation.

LEMMA 4.9. Let $R$ have FGSP and possess a semiprimary classical right quotient ring $Q$. Then $f_{2} \in R_{2}$; so $e_{2} \in R_{2}$.

Proof. Let $M=R_{2} \cap f_{2} Q_{2}$. Then it follows from Lemma 3.1 and the remarks following the lemma that $M=f_{2} R_{2}$ where $f_{2} \in R_{2}$.

Lemma 4.10. Let $R$ have FGSP and possess a semiprimary classical right quotient ring $Q$. Then $e_{2} R_{2} e_{2}$ is semiprime and is right finite dimensional.

Proof. First note that $f_{2} R_{2}$ is a maximal two-sided ideal of $L^{*}\left(R_{2}\right)$ whose left annihilator is essential is a right ideal of $R$. For suppose $f_{2} R_{2} \subseteq M$ where $M$ is a two-sided ideal of $L^{*}\left(R_{2}\right)$ and $H=l_{R_{2}}(M)$ is essential as a right ideal of $R_{2}$. By Lemma 3.1, $M=$ $g R_{2}$ for $g$ an idempotent element of $R_{2}$. Then $M Q_{2}=g Q_{2}$ is a twosided ideal of $L^{*}\left(Q_{2}\right)$ whose left annihilator contains $H$. Hence $g Q_{2}$ has a right essential left annihilator in $Q_{2}$. Therefore $e_{2} g=0$; so since $f_{2} R_{2} \subseteq M, g \in f_{2} R_{2}$, and $M=f_{2} R_{2}$.

Claim $e_{2} R_{2} e_{2}$ is semiprime. Let $I$ be a nilpotent ideal of $e_{2} R_{2} e_{2}$. If $P$ is the closure of $I$ in $e_{2} R_{2} e_{2}$, then $P$ is a two-sided ideal of $L^{*}\left(e_{2} R_{2} e_{2}\right)$ whose left annihilator is right essential in $e_{2} R_{2} e_{2}$ by [17, Lemma A]. Then it follows that

$$
\left[\begin{array}{lc}
P & 0 \\
f_{2} R_{2} e_{2} & f_{2} R_{2} f_{2}
\end{array}\right]
$$

is a two-sided ideal of $L^{*}\left(R_{2}\right)$ whose left annihilator is right essential in $R_{2}$. This forces $I$ to be zero as $f_{2} R_{2}$ is the maximal twosided ideal of $R_{2}$ with this property. Thus $e_{2} R_{2}$ is semiprime.

Since $Q$ is an essential extension of $R$, it follows that $R \cap e Q$ is essential in $e Q$. Therefore $R \cap\left(0 \times e_{2} Q_{2}\right)$ is essential in $0 \times e_{2} Q e_{2}$; so $e_{2} R_{2} e_{2}$ is essential in $e_{2} Q_{2} e_{2}$. Thus $e_{2} Q_{2} e_{2}=S^{0}\left(e_{2} R_{2} e_{2}\right)$ and $e_{2} R_{2} e_{2}$ is finite dimensional since $e_{2} Q_{2} e_{2}$ is semisimple Artinian.

THEOREM 4.11. A right nonsingular ring $R$ which possesses a semiprimary classical right quotient ring has FGSP if and only if $R=R_{1} \times R_{2}$ where $R_{1}$ has essential socle, $R_{2}$ has zero socle and both $R_{1}$ and $R_{2}$ have FGSP with semiprimary classical right quotient rings.

Proof. Let $R$ have FGSP. By the choice of $e_{1}$ and $f_{1}, R_{1} e_{1}$ is 
essential as a right ideal of $R_{1}$ and $R_{1}$ has FGSP by Lemma 4.1. Since $R_{1}$ has essential socle, the techniques of $\S 2$ can be applied to show that

$$
R_{1}=\left[\begin{array}{cc}
e_{1} R_{1} e_{1} & 0 \\
f_{1} R_{1} e_{1} & f_{1} R_{1} f_{1}
\end{array}\right]=\left[\begin{array}{cc}
A_{1} & 0 \\
B_{1} & C_{1}
\end{array}\right]
$$

where $A_{1}$ is semisimple Artinian and $C_{1}$ is a semihereditary subring of $T=\operatorname{End}_{A}(B)$ such that $T_{C}$ is flat.

Since $R_{2}$ has FGSP by Lemma 4.1, we have shown in Lemmas 4.9 and 4.10 that $R_{2}=\left[\begin{array}{cc}A_{2} & 0 \\ B_{2} & C_{2}\end{array}\right]$ where $A_{2}=e_{2} R_{2} e_{2}$ is a right finite dimensional semiprime ring. By a slight modification of the proof of Lemma 3.3, we see that $C_{2}=f_{2} R_{2} f_{2}$ is a semihereditary ring possessing a two-sided maximal quotient ring $T_{2}$ which is right $C_{2^{-}}$ flat.

The proof of Lemma 4.4 can be modified to yield that $E_{1}^{\prime}$ is a direct summand of $C_{1}$ and $E_{2}^{\prime}=C_{2}$.

$E_{2}^{\prime}=C_{2}=f_{2} R_{2} f_{2}$; so $f_{2} \in R$. Thus by [18, Theorem A] $f_{2} Q f_{2}=$ $f_{2} Q_{2} f_{2}$ is the semiprimary classical right quotient ring of $C_{2}$. Also $f_{2} Q_{2} f_{2} \subseteq f_{2}\left(S^{0} R\right) f_{2}$; so by Lemmas 2.6 and $4.10, R_{2}$ has a semiprimary classical right quotient ring $Q_{2}$. Therefore $R_{2}$ is right finite dimensional by Proposition 3.5. Now it follows that

$$
R_{1}=\left[\begin{array}{ccc}
A_{11} & 0 & 0 \\
A_{21} & A_{22} & 0 \\
A_{31} & A_{32} & A_{33}
\end{array}\right], \quad E_{1}=\left[\begin{array}{ccc}
A_{11} & 0 & 0 \\
A_{21} & 0 & 0 \\
A_{31} & A_{32} & A_{33}
\end{array}\right]
$$

and

$$
R_{2}=\left[\begin{array}{cc}
A_{2} & 0 \\
B_{2} & C_{2}
\end{array}\right], \quad E_{2}=\left[\begin{array}{cc}
X & 0 \\
B_{2} & C_{2}
\end{array}\right]
$$

where $A_{\imath j}, A_{2}, B_{2}, C_{2}$, and $X$ are as in Theorem 4.5.

If $R$ is not the direct product of $R_{1}$ and $R_{2}, X \neq A_{2}$. If

$$
\left(\left[\begin{array}{ccc}
a_{11} & 0 & 0 \\
a_{21} & a_{22} & 0 \\
a_{31} & a_{32} & a_{33}
\end{array}\right],\left[\begin{array}{ll}
0 & 0 \\
b & c
\end{array}\right]\right) \in R
$$

where $a_{i j} \in A_{i j}, b \in B_{2}$ and $c \in C_{2}$, then $a_{22}=0$. Claim

$$
P=\left(\left[\begin{array}{ccc}
0 & 0 & 0 \\
A_{21} & 0 & 0 \\
A_{31} & A_{32} & N\left(A_{33}\right)
\end{array}\right], \quad\left[\begin{array}{cc}
0 & 0 \\
B_{2} & N\left(C_{2}\right)
\end{array}\right]\right)
$$

is the prime radical of $R$. $P$ is nilpotent; so $P \subseteq N(R)$. Let 


$$
U=\left(\left[\begin{array}{lll}
0 & 0 & 0 \\
0 & I & 0 \\
0 & 0 & 0
\end{array}\right], \quad\left[\begin{array}{ll}
J & 0 \\
0 & 0
\end{array}\right]\right),
$$

and suppose that $U \subset N(R) . N(R)$ is nilpotent so $U^{n}=0$ for some positive integer $n$. Thus $I^{n}=0$ and $J^{n}=0$. Since $A_{2}$ is semiprime, then $J=0$. Then it follows that $I=0$. From this it is immediate that $P=N(R)$.

Since $A_{2}$ possesses a semisimple classical right quotient ring and $X \neq A_{2}$ is essential as a right ideal of $A_{2}$, there is a regular element of $d$ of $A_{2}$ such that $d \in X$. Consider the element

$$
x=\left(\left[\begin{array}{lll}
1 & 0 & 0 \\
0 & 0 & 0 \\
0 & 0 & 1
\end{array}\right], \quad\left[\begin{array}{ll}
d & 0 \\
0 & 1
\end{array}\right]\right) \in R
$$

and let $\bar{x}=x+N(R)$. Now $\bar{x}$ is a regular element of $R / N(R)$. For if

$$
\bar{y}=\left(\left[\begin{array}{ccc}
a_{11} & 0 & 0 \\
0 & a_{22} & 0 \\
0 & 0 & a_{33}
\end{array}\right], \quad\left[\begin{array}{ll}
a & 0 \\
0 & c
\end{array}\right]\right)+N(R)
$$

is such that $\bar{x} \bar{y}=0$, then $a_{11}=0, a_{33} \in N\left(A_{33}\right), c \in N\left(C_{2}\right)$ and $d a=0$. Since $d$ is a regular element of $A_{2}, a=0$. Hence $a_{22}=0$; so $\bar{y}=\overline{0}$. Similarly $\bar{y} \bar{x}=\overline{0}$ implies $\bar{y}=\overline{0}$. By the proof of [29, Theorem 2.12], $x$ must be a regular element of $R$. However if $a_{21} \neq 0$ is in $A_{21}$,

$$
\left(\left[\begin{array}{lll}
1 & 0 & 0 \\
0 & 0 & 0 \\
0 & 0 & 1
\end{array}\right],\left[\begin{array}{ll}
d & 0 \\
0 & 1
\end{array}\right]\right)\left(\left[\begin{array}{ccc}
0 & 0 & 0 \\
a_{21} & 0 & 0 \\
0 & 0 & 0
\end{array}\right], \quad\left[\begin{array}{ll}
0 & 0 \\
0 & 0
\end{array}\right]\right)=0,
$$

and $x$ is not a regular element of $R$. This contradiction yields that $X=A_{2}$ and $R=R_{1} \times R_{2}$.

The converse is obvious.

REMARK. Another situation in which the essential product of Theorem 4.5 is actually a direct product occurs when $A_{2}$ is commutative. Then $A_{2}$ is a direct product of Prüfer domains. If $X$ is as in Theorem 4.5, then there is a nonzero divisor $d \in X$. If $X=d A_{2}$, then $X$ is a finitely generated idempotent ideal of $A_{2}$. But it is well known that such an ideal is a direct summand of $A_{2}$ which is impossible. If $d A_{2} \subseteq X$, then $X / d_{2} A_{2}$ is a direct summand of $A_{2} /$ $d_{2} A_{2}$. But again this forces $X$ to be finitely generated which is impossible. Thus $X=A_{2}$ and the product is direct. 
5. Applications to rings with SP and BSP. Let $M$ be a singular $R$-module and $I$ an essential right ideal of $R$. We say that $M$ has bounded order $I$ if $M$ can be embedded in a module which has a set of generators all annihilated by $I . M$ has bounded order if $M$ has bounded order for some essential right ideal $I$ of $R$. A ring $R$ has bounded splitting property (BSP), if $Z(M)$ is a direct summand of $M$ for every right $R$-module $M$ such that $Z(M)$ has bounded order.

We now make some observations on how the results and techniques developed in this paper can be applied to rings with SP and BSP. If a ring $R$ has BSP or SP, then $R$ has FGSP. Also by the proof of [16, Theorem 5.3] if $R$ has BSP or SP and $H$ is a twosided ideal of $R$ which is essential as a right ideal, then $R / H$ is right perfect.

Let $R$ be a right nonsingular right finite dimensional ring with zero socle. If $R$ has BSP, then $R$ has FGSP; so $R=\left[\begin{array}{ll}A & 0 \\ B & C\end{array}\right]$ where $A, B$, and $C$ satisfy the conditions of Theorem 3.6. Since $\left[\begin{array}{cc}A & 0 \\ B & C\end{array}\right]$ is essential in $R_{R}$, then $C$ is a semiprimary ring. Hence by Proposition 3.11, $B_{A}$ is injective. Thus we have the following result.

THEOREM 5.1. Let $R$ be a right nonsingular right finite dimensional ring with zero socle and BSP. Then $R$ is isomorphic to

$$
\left[\begin{array}{ll}
A & 0 \\
B & C
\end{array}\right]
$$

where

(1) A is a semiprime right nonsingular right finite dimensional ring with zero socle and $B S P$;

(2) $B$ is a $C-A$ bimodule which is a finite dimensional nonsingular injective A-module;

(3) $C$ is a semiprimary ring with a two-sided maximal quotient ring $T=\operatorname{End}_{A}(B)$.

Conversely, any such matrix ring is a right nonsingular right finite dimensional ring with zero socle and has BSP.

Proof. We only need to prove the converse. But this follows by a slight modification of the arguments used to prove $[17$, Theorem 6].

Thus the $\operatorname{ring}\left[\begin{array}{ll}\boldsymbol{Z} & 0 \\ \boldsymbol{Q} & \boldsymbol{Z}\end{array}\right]$ has FGSP by Theorem 3.6 and Corollary 
3.13 whereas $\left[\begin{array}{ll}\boldsymbol{Z} & 0 \\ \boldsymbol{Q} & \boldsymbol{Q}\end{array}\right]$ has BSP. $\boldsymbol{Z}$ denotes the integers and $\boldsymbol{Q}$ the rational numbers.

If $R$ has essential socle and is either right finite dimensional or a right order in a semiprimary classical right quotient ring, then $\mathrm{BSP}$ is equivalent to SP and the structure of these rings are given in [8].

We now turn to the problem of expressing a right nonsingular ring $R$ with $\mathrm{BSP}$ or SP as an essential product of $R_{1}$ and $R_{2}$ where $R_{1}$ has essential socle and $R_{2}$ has zero socle. Two conditions were critical in order to obtain Theorem 4.5. First of all, each $R_{i}$ was isomorphic to a formal triangular matrix ring $\left[\begin{array}{ll}A & 0 \\ B & C\end{array}\right]$ where $\left[\begin{array}{ll}A & 0 \\ B & 0\end{array}\right]$ is an essential right ideal. Secondly, the $E_{i}$ 's must satisfy Lemma 4.3. The second condition holds if $R$ has SP or BSP. If $R$ has $S P$ with no infinite sets of orthogonal idempotents in its socle, then $R_{1}$ and $R_{2}$ has the desired triangular form by [8, Theorem 3.5] and [17, Theorem 7]. Furthermore, if $R$ is right finite dimensional and has BSP, $R_{1}$ and $R_{2}$ have the required triangular form by Theorem 2.5 (or [8, Theorem 3.5]) and Theorem 2.6. Thus in these cases a theorem similar to Theorem 4.5 can be proven.

ACKNOWLEDGMENT. The authors would like to express their gratitude to Mark L. Teply and to the referee for a number of helpful suggestions.

\section{REFERENCES}

1. V. Camillo, $A$ note on semihereditary rings, Arch. Math. (Basel), 24 (1973), 142143.

2. H. Cartan and S. Eilenberg, Homological Algebra, Princeton University Press, Princeton, N. J., 1956

3. V. C. Cateforis, Flat regular quotient rings, Trans. Amer. Math. Soc., 138 (1969), 241-249.

4. —- On regular self-injective rings, Pacific J. Math., 30 (1969), 39-45.

5. - Two-sided semisimple maximal quotient rings, Trans. Amer. Math. Soc., 149 (1970), 339-349.

6. V. C. Cateforis and F. L. Sandomierski, The singular submodule splits off, J. Algebra, 10 (1968), 149-165.

7. J. H. Cozzens, Homological properties of the ring of differentiable polynomials, Bull. Amer. Math. Soc., 76 (1970), 75-79.

8. J. Fuelberth and J. Kuzmanovich, On the structure of splitting rings, Comm. in Algebra, 3 (1975), 913-949.

9. —_ Split subdirect products and piecewise domains, Canad. J. Math., 28 (1976), 408-419.

10. The structure of semiprimary and Northerian hereditary rings, Trans. Amer. Math. Soc., 212 (1975), 83-111.

11. J. D. Fuelberth and M. L. Teply, The singular submodule of a finitely generated module splits off, Pacific J. Math., 40 (1972), 73-82. 
12. O. Goldman, Rings and modules of quotients, J. Algebra, 13 (1969), 10-47.

13. K. R. Goodearl, Essential products of nonsingular rings, Pacific J. Math., 45 (1973), 495-505.

14. Localization and splitting in hereditary Northerian prime rings, Pacific. J. Math., 53 (1974), 137-151.

15. — Ring theory, Nonsingular Rings and Modules, Marcel Dekker, Inc., New York and Basel, 1976.

16. - Singular torsion and splitting properties, Memoirs Amer. Math. Soc., 124 (1972).

17. - Triangular representation of splitting rings, Trans. Amer. Math. Soc., 185 (1973), 271-285.

18. R. Gordon, Classical quotient rings of PWD's, Proc. Amer. Math. Soc., 36 (1972), $39-46$.

19. - Rings faithfully represented on their left socle, J. Algebra, 7 (1967), 303-342.

20. - Rings in which minimal left ideals are projective, Pacific. J. Math., 31 (1969), 679-692.

21. R. Gordon and L. W. Small, Piecewise domains, J. Algebra, 23 (1972), 553-564.

22. C. U. Jensen, Arithmetical rings, Acta Math. Acad. Sci. Hung., 17 (1966), 115123.

23. S. Jøndrup, On finitely generated flat modules II, Math. Scand., 27 (1970), 105112.

24. B. L. Osofsky, Cyclic injective modules of full linear rings, Proc. Amer. Math. Soc., 17 (1966), 247-253.

25. J. C. Robson, Idealizers and hereditary Northerian prime rings, J. Algebra, 22 (1972), 45-81.

26. F. L. Sandomierski, Semisimple maximal quotient rings, Trans. Amer. Math. Soc., 128 (1967), 112-120.

27. L. Silver, Noncommutative localizations and applications, J. Algebra, 7 (1967), $44-76$.

28. L. W. Small, Semihereditary rings, Bull. Amer. Math. Soc., 73 (1967), 656-658.

29. - Orders in Artinian rings, J. Algebra, 4 (1966), 13-41.

30. B. Stenstrom, Rings of Quotients, Die Grundlehren der mathematischen Wissenschaften, 217, Springer-Verlag, New York-Heidelberg-Berlin, 1975.

Received June 15, 1977 and in revised form March 29, 1978. The first author was partially supported by a grant from the Research and Publication Committee at the University of Northern Colorado.

University of Northern Colorado

Greeley, CO 80639

AND

WAKe Forest University

Winston-Salem, NC 27109 


\section{PACIFIC JOURNAL OF MATHEMATICS}

\section{EDITORS}

RICHARD ARENS (Managing Editor)

University of California

Los Angeles, CA 90024

Charles W. Curtis

University of Oregon

Eugene, OR 97403

C. C. MOORE

University of California

Berkeley, CA 94720
J. DUGUNDJI

Department of Mathematics

University of Southern California

Los Angeles, CA 90007

R. FINN and J. MILGRAM

Stanford University

Stanford, CA 94305

\section{ASSOCIATE EDITORS}

\section{E. F. BeCKENBACH}

B. H. NeumanN

F. WOLF

K. YoSHIDA

\section{SUPPORTING INSTITUTIONS}

\author{
UNIVERSITY OF SOUTHERN CALIFORNIA \\ STANFORD UNIVERSITY \\ UNIVERSITY OF HAWAII \\ UNIVERSITY OF TOKYO \\ UNIVERSITY OF UTAH \\ WASHINGTON STATE UNIVERSITY \\ UNIVERSITY OF WASHINGTON
}

The Supporting Institutions listed above contribute to the cost of publication of this Journal, but they are not owners or publishers and have no responsibility for its content or policies.

Mathematical papers intended for publication in the Pacific Journal of Mathematics should be in typed form or offset-reproduced, (not dittoed), double spaced with large margins. Please do not use built up fractions in the text of the manuscript. However, you may use them in the displayed equations. Underline Greek letters in red, German in green, and script in blue. The first paragraph or two must be capable of being used separately as a synopsis of the entire paper. Items of the bibliography should not be cited there unless absolutely necessary, in which case they must be identified by author and journal, rather than by item number. Manuscripts, in triplicate, may be sent to any one of the editors. Please classify according to the scheme of Math. Reviews, Index to Vol. 39. All other communications should be addressed to the managing editor, or Elaine Barth, University of California, Los Angeles, California, 90024.

50 reprints to each author are provided free for each article, only if page charges have been substantially paid. Additional copies may be obtained at cost in multiples of 50 .

The Pacific Journal of Mathematics is issued monthly as of January 1966. Regular subscription rate: $\$ 72.00$ a year (6 Vols., 12 issues). Special rate: $\$ 36.00$ a year to individual members of supporting institutions.

Subscriptions, orders for numbers issued in the last three calendar years, and changes of address should be sent to Pacific Journal of Mathematics, P.O. Box 969, Carmel Valley, CA 93924, U.S.A. Older back numbers obtainable from Kraus Periodicals Co., Route 100, Millwood, NY 10546.

PUBLISHED BY PACIFIC JOURNAL OF MATHEMATICS, A NON-PROFIT CORPORATION

Printed at Kokusai Bunken Insatsusha (International Academic Printing Co., Ltd.). 8-8, 3-chome, Takadanobaba, Shinjuku-ku, Tokyo 160, Japan. 


\section{Pacific Journal of Mathematics}

\section{Vol. 80, No. $2 \quad$ October, 1979}

K. Adachi, On the multiplicative Cousin problems for $N^{p}(D) \ldots \ldots \ldots \ldots 297$

Howard Banilower, Isomorphisms and simultaneous extensions in $C(S) \ldots 305$

B. R. Bhonsle and R. A. Prabhu, An inversion formula for a distributional

finite-Hankel-Laplace transformation ................... 313

Douglas S. Bridges, Connectivity properties of metric spaces.......... 325

John Patton Burgess, A selection theorem for group actions ........... 333

Carl Claudius Cowen, Commutants and the operator equations

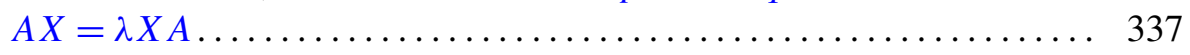

Thomas Curtis Craven, Characterizing reduced Witt rings. II .......... 341

J. Csima, Embedding partial idempotent d-ary quasigroups ............ 351

Sheldon Davis, A cushioning-type weak covering property ............ 359

Micheal Neal Dyer, Nonminimal roots in homotopy trees ............. 371

John Erik Fornaess, Plurisubharmonic defining functions ........... 381

John Fuelberth and James J. Kuzmanovich, On the structure of finitely

generated splitting rings .......................... 389

Irving Leonard Glicksberg, Boundary continuity of some holomorphic

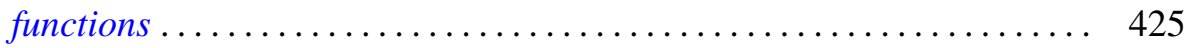

Frank Harary and Robert William Robinson, Generalized Ramsey theory.

IX. Isomorphic factorizations. IV. Isomorphic Ramsey numbers .......

Frank Harary and Allen John Carl Schwenk, The spectral approach to determining the number of walks in a graph...........

David Kent Harrison, Double coset and orbit spaces ..... . .

Shiro Ishikawa, Common fixed points and iteration of commuting

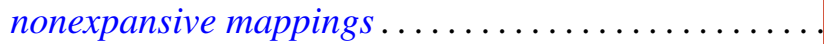

Philip G. Laird, On characterizations of exponential polynomials ........ 503

Y. C. Lee, A Witt's theorem for unimodular lattices ...........

Teck Cheong Lim, On common fixed point sets of commutative

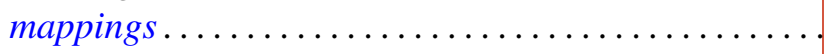

R. S. Pathak, On the Meijer transform of generalized functions ...

T. S. Ravisankar and U. S. Shukla, Structure of $\Gamma$-rings . . .

Olaf von Grudzinski, Examples of solvable and nonsolvable convolution

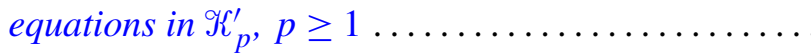

